\title{
A Review of the Torsional Split Hopkinson Bar
}

\author{
Xiao Yu, ${ }^{1}$ Li Chen $\mathbb{D}^{1,2}$ Qin Fang, ${ }^{1}$ Xiquan Jiang, ${ }^{1}$ and Yongkang Zhou ${ }^{1}$ \\ ${ }^{1}$ Army Engineering University of PLA, State Key Laboratory of Disaster Prevention \& Mitigation of Explosion \& Impact, \\ Nanjing 210007, China \\ ${ }^{2}$ School of Civil Engineering, Southeast University, Nanjing 210096, China \\ Correspondence should be addressed to Li Chen; chenli1360@qq.com
}

Received 14 June 2018; Accepted 14 August 2018; Published 10 October 2018

Academic Editor: Venu G. M. Annamdas

Copyright ( 12018 Xiao Yu et al. This is an open access article distributed under the Creative Commons Attribution License, which permits unrestricted use, distribution, and reproduction in any medium, provided the original work is properly cited.

\begin{abstract}
Mechanical behavior of materials at medium and high strain rates $\left(10^{1} \sim 10^{4} \mathrm{~s}^{-1}\right)$ is the foundation of developing mechanical theories, building material models, and promoting engineering design and construction. The torsional split Hopkinson bar (TSHB) is an effective experimental technique for measuring the pure shear mechanical properties of materials at high strain rates. In this study, the state-of-the-art in TSHB experimental technique is presented. Five typical types of TSHB loading mechanisms, i.e., prestored energy loading, explosive loading, direct impact loading, flywheel loading, and electromagnetic loading, were systematically reviewed. The TSHB fundamentals were outlined, which include elementary components, basic assumptions, working principles, the pulse shaping technique, specimen design, and the single-pulse loading technique. In addition, the combined loading and high/low temperature experimental techniques, which were developed based on TSHB, were also discussed in detail. Nearly all necessary elements for conducting a TSHB experiment and analyzing the experimental data were provided. Some research directions should be further pursued, such as extending the range of applicable materials and developing the combined loading techniques.
\end{abstract}

\section{Introduction}

As early as the 19th century, scientists gradually realized that the mechanical properties of materials under dynamic loads were significantly different from those under static loads, which led to the development of experimental techniques on testing materials under high strain rates. Thereafter, during World War II, the strong military demand rapidly propelled studies of dynamic mechanical properties on materials. After World War II, most countries entered a period of rapid recovery and development, and mechanical properties and structural responses under impact loads became more important in nearly all areas of engineering, such as mechanical machining, building construction, high-speed travel, and aircraft and spacecraft designs [1]. In addition, more frequent occurrences of natural hazards and explosion and impact accidents, such as earthquakes, terrorist explosions, gas explosions, and vehicle-to-ship collisions, allowed engineering structures prone to impact loads during the structures' service time, which also greatly promoted studies on the dynamic behavior of materials and structures. With popularization of the computer and algorithm progress, numerical simulations became more popular and important in engineering design, prediction, and evaluation. The fundamental elements of numerical approaches are the right material modes and failure algorithms under high strain rates, both of which are built based on reliable and validated experimental results.

According to the strain rate of materials, mechanical experiments can be roughly divided into three ranges, i.e., creep experiment, quasistatic experiment and dynamic experiment. The strain rate of the creep experiment is $10^{-8} \sim 10^{-6} \mathrm{~s}^{-1}$ and the strain rate of the quasistatic experiment is $10^{-5} \sim 10^{-1} \mathrm{~s}^{-1}$. The dynamic experiment can be further divided into medium strain rate experiment, high strain rate experiment, and ultrahigh strain rate experiment, which correspond to the strain rates of $10^{-1} \sim 10^{1} \mathrm{~s}^{-1}$, $10^{2} \sim 10^{4} \mathrm{~s}^{-1}$, and $>10^{4} \mathrm{~s}^{-1}$, respectively. There are many mature experimental techniques for compression and tension at a high strain rate. For high strain rate compressive experiments, drop weight [2], split Hopkinson pressure bar (SHPB) [3], and gas guns [4] are commonly used. For tensile 
experiments, expanding ring [5, 6] and split Hopkinson tensile bar (SHTB) [7, 8] are commonly used. In addition, SHPB was also used for split [9], spalling [10], and threepoint bending [11] experiments to measure the approximate tensile behaviors of materials under high strain rates. However, the stress states of the specimens were complex in these experimental conditions, and the stress states of the specimens were not in an axial tensile state.

The dynamic shear experimental technique can be divided into two main types: compressive-shear loading apparatus and torsional loading apparatus.

The compressive-shear loading apparatus mainly includes a series of universal testing machines produced by the MTS Corporation and the punch-shear technique based on $\mathrm{SHPB}$. The universal testing machines are generally applicable to materials under low to medium strain rates but are not applicable for high strain rates. The punch-shear technique, which uses SHPB to test the specially designed specimen, is commonly used for materials at medium to high strain rates. Using SHPB, Dowling et al. [12] designed a punch-shear experiment. The transmission bar was designed as a tube with an inner diameter larger than the diameter of the incident bar, which allowed the incident bar to slide into the transmission bar. A thin-cylinder specimen was set between the incident bar and transmission bar. When the incident wave was loaded on the specimen, the narrow-ring area between the area in contact with the incident bar and the area in contact with the transmission bar was subjected to shear. The test strain rate was up to $10^{4} \mathrm{~s}^{-1}$. Harding and Huddart [13] modified the specimen. A double-notch shear specimen was designed, which contains a flange and groove. The flange diameter was the same as that of the incident bar, and the outer diameter of the groove was the same as that of the transmission bar. Thus, the connecting area between the flange and groove was under shear stress. The strain rate of the specimen reached $10^{4} \mathrm{~s}^{-1}$. Klepaczko [14] modified the double-notch shear specimen and directly impacted it with the striker, which shortened the rising time of the incident wave, and the strain rate reached $10^{5} \mathrm{~s}^{-1}$. After the 1970s, some other techniques started to be used to conduct dynamic pressure-shear experiments, such as plate impact experiments using light gas and electromagnetic guns [15-17] and shock wave propagation experiments on anisotropic quartz loaded by explosives [18]. However, although researchers have used a variety of methods to study the dynamic shear of materials, the defect of the non-torsional loading method remains obvious: the stress on the shear surface of the specimen cannot be guaranteed during the entire experimental process. Thus, the specimen is not completely in a state of pure shear, which can lead to great measurement errors or even the wrong conclusions.

The torsional loading apparatus that is most commonly used is the torsional split Hopkinson bar (TSHB). TSHB was first developed by Baker and Yew [19] in 1966. The specimen was designed as a thin-walled tube to maintain the pure shear state. The stress equilibrium at both ends of the specimen guarantees that the specimen is uniformly sheared. Compared with the non-torsional loading apparatus, the
TSHB has obvious advantages in terms of precision. In addition, the TSHB avoids some of the problems encountered by the SHPB. Propagation of the elastic torsional wave can avoid the dispersion effect in bars, thin-walled tubular specimens can minimize the inertial effect, and no radial displacement between the specimen and the bars can avoid the frictional effect. Therefore, the TSHB is a good choice for testing the dynamic shear behaviors of materials, which can improve the accuracy of the experimental results and save a large amount of time correcting experimental data.

The TSHB has been developed by many researchers over the past decades [20-24]. Although there are handbook [25], book [3], and reviews [26-29] detailed discussed the development and application of the split Hopkinson bar technique, a specialized review of the TSHB is needed. In this review, the development history of the TSHB is presented. Some critical theories and techniques, such as the basic composition, fundamental assumptions, working principle, pulse shaping, specimen design, and single pulse loading technology are presented in detail. By analyzing the deficiencies in the existing TSHB experimental technique, several issues worthy of research and exploration are proposed.

\section{Historical Background}

After developed by Kolsky [30] in 1949, the SHPB has been widely applied to test the dynamic behavior of materials under compression [31-33]. Kolsky used a thin specimen, whose length was short enough to ignore its internal stress wave propagation process. Kolsky placed the specimen between two long elastic bars, which allowed for calculation of the exact stress histories on both ends of the specimen by time-shifting the elementary elastic waves recorded in the bars. This ingenious design successfully decoupled the stress wave propagation effect and strain rate effect in the specimen. Inspired by this idea, Baker and Yew [19] combined the Hopkinson bar technique with a specially designed loading mechanism on a torsional wave in 1966, which is called the torsional split Hopkinson bar (TSHB) technique.

Fifty years after the development, TSHB has been applied to test the high strain rate mechanical behaviors of various materials, such as steel [34], lead [35], titanium [36], aluminum [37], OFHC copper [38], and tungsten heavy alloys [39]. According to the loading mechanisms, TSHB can be roughly divided into five types: prestored energy loading [19], explosive loading [40], direct impact loading [41], flywheel loading [42], and electromagnetic loading [43].

\subsection{Prestored Energy Loading TSHB}

2.1.1. Pioneering Work of Baker and Yew on TSHB. In 1966, Baker and Yew [19] designed the first TSHB in the world, which is shown in Figure 1. The apparatus mainly contains an incident bar and a transmission bar, which are both steel tubes. One end of the incident bar was clamped by a lathe chuck and the middle of the incident bar was fixed by a clamp. The torsional strain energy was stored in the portion of the incident bar between the lathe chuck and 


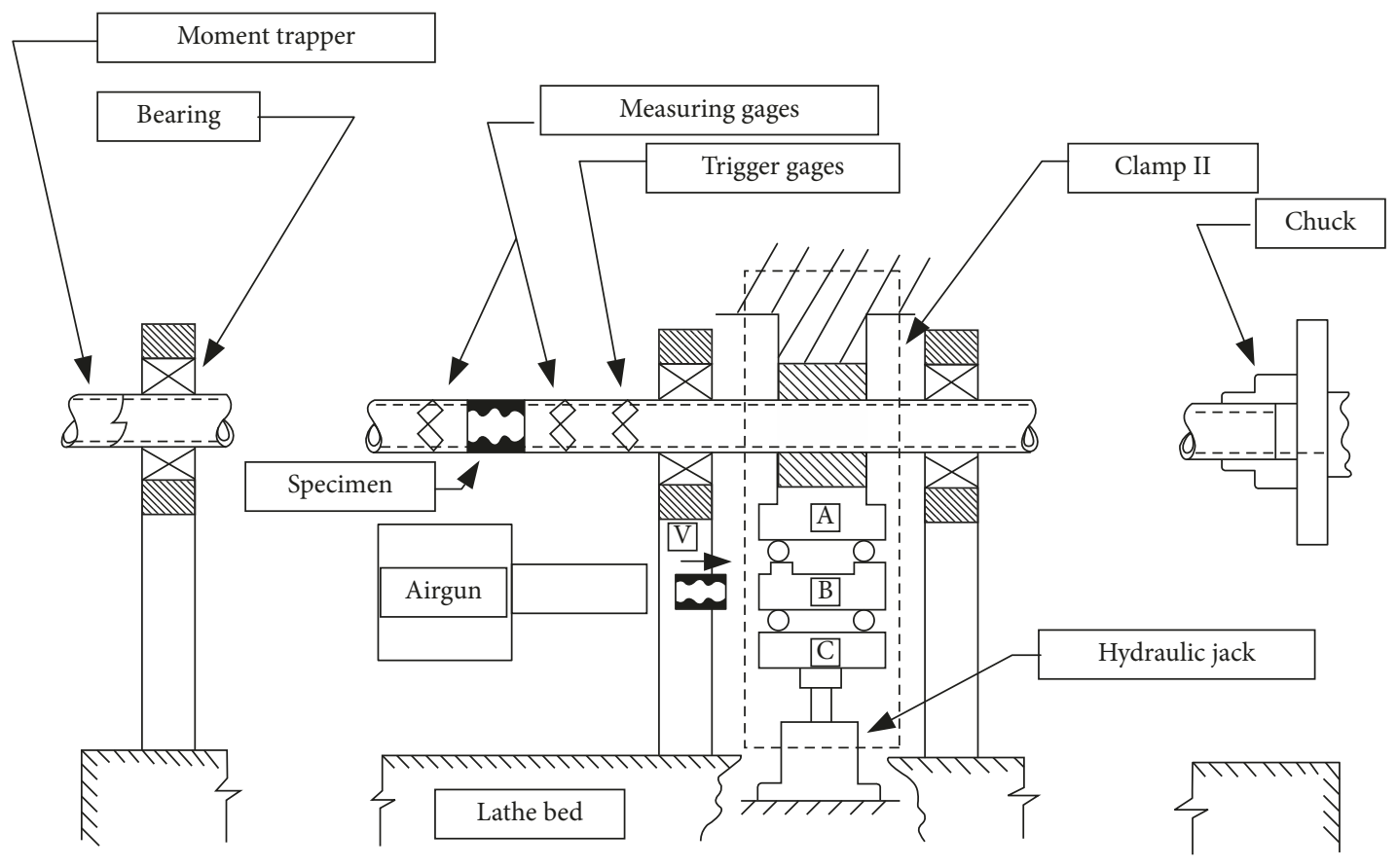

FIGURE 1: Sketch of the TSHB designed by Baker and Yew [19].

clamp by turning the lathe head through an angle. When the bar was twisted to the predetermined angle, the clamp was suddenly released, and then, the torsional stress wave was instantly generated. The clamp is crucial for this loading mechanism. The clamp can directly influence the rising time of the torsional wave, which influences the strain rate of the specimen. Figure 2 shows the clamp detail, which is released by knocking out plane $\mathrm{B}$, and the shortest rising time of the torsional wave is approximately $30 \mu \mathrm{s}$. In addition, the clamp mechanism relates to the success rate of the experiments. The holding force of the clamp cannot be too large to ensure that the incident bar remains in its elastic deformation region, and the force cannot be too small to ensure no slippage occurs between the incident bar and the clamp.

The amplitude of the torsional wave can be controlled by adjusting the twisting angle of the incident bar. If the required torsional wave amplitude exceeds the yield strength of the bar material, an incident bar with a higher yield strength needs to be used. The duration of the torsional wave can be controlled by adjusting the length of the incident bar clamped portion, which is between the lathe chuck and clamp. The cylindrical tube copper specimen was connected to the incident bar and transmission bar by welding, which can provide a tight connection. The torsional waves were measured by strain gauges pasted on the bar surfaces. The pasted direction of the strain gauges was 45 degrees to the axial direction of the bars.

2.1.2. Development of the Clamp. In 1970, Campbell and Dowling [44] proposed a new type of clamp. As shown in Figure 3, the incident bar was placed between bridge piece $\mathrm{A}$ and cradle $B$, which were fastened by a high-tensile steel wire. The clamp force can be adjusted by controlling the

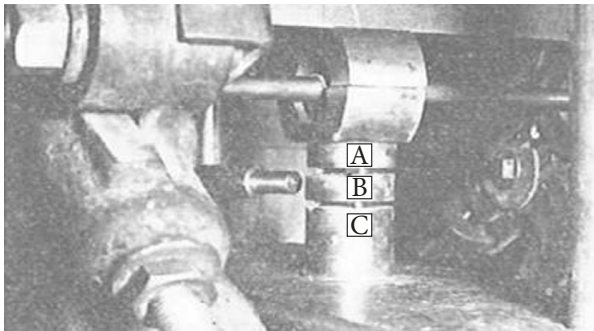

Figure 2: Details of the clamp [19].

tightness of bolt I, and the force value can be measured by dynamometer $\mathrm{D}$. The clamp was released by nicking the wire at its middle point $\mathrm{K}$. This design is more precise and easier to control than that of Baker and Yew, but the rising time of the torsion pulse is slightly longer, which is up to $50 \mu \mathrm{s}$.

In 1972, Lewis and Campbell [45] designed a new clamp, which is shown in Figure 4. A tapered flange was machined in the middle of the incident bar, which was cemented to a fixed holding ring by an epoxy adhesive. The adhesive provided a strong clamp force. The adhesive layer broke instantaneously when the loading force exceeded the strength of the adhesive, and then, the torsional pulse was generated. The rising time of the torsional wave generated by this method is $20 \sim 25 \mu \mathrm{s}$. This clamp can release a larger amplitude of torsional wave than the previous clamps. In addition, Lewis and Campbell [45] used an adhesive bonding to replace the welding between the specimen and bars, which increased the convenience of the experiments. However, this type of clamp contains intrinsic deficiencies. The asymmetric development of the fracture joints of the epoxy adhesive leads to the generation of bending waves, which challenges the pure shear state of the specimen and makes it difficult to measure the torsional waves in the bars. In addition, 


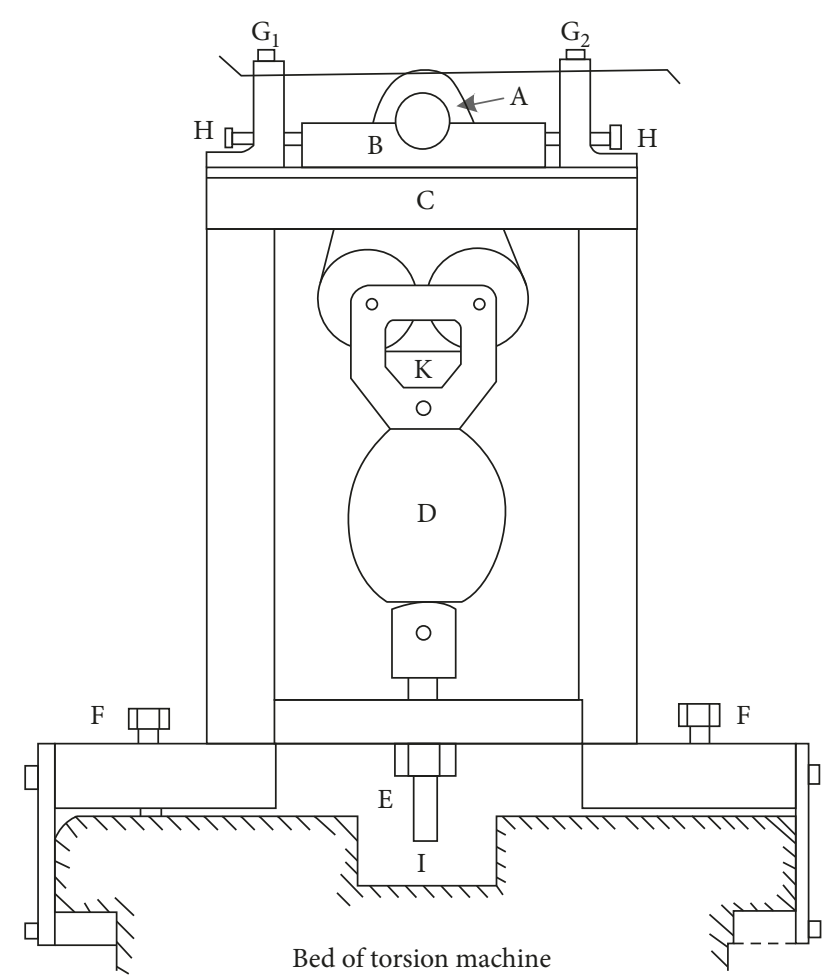

Figure 3: The clamp designed by Campbell and Dowling [44].

unpredictable fracturing of the epoxy adhesive makes the prestored energy difficult to control, which creates difficulties in precisely controlling the amplitude of the torsional wave.

In 1972, Nicholas and Lawson [20] introduced a clamp with a notched pin. The prestored torque is released by a further tightening of the clamp until the notched pin breaks. This type of clamp was reported frequently in later investigations [21, 46, 47]. Hartley et al. [48] modified the clamp and introduced the detailed structure of the clamp, which is shown in Figure 5. This clamp consists of two arms. The top of two arms are held together by a notched pin. One side of arms at the bottom is hinged to a fixed peg, and the other side of arms at the bottom is hinged to a sliding peg. A hydraulic ram compresses the lower parts of the arms to tighten the clamp. The clamp is released by further increasing the hydraulic pressure, which eventually induced fracturing in the notched pin at the top of the clamp. The rising time of the pulse generated by this clamp can reach $40 \mu \mathrm{s}$. This type of clamp has the characteristics of good reproducibility and a relatively simple manufacturing process, which makes this clamp widely used in TSHB experiments.

Prestored energy loading has been the most commonly used technique in the past decades since the TSHB was originally invented, but this type of loading mechanism has certain deficiencies. Due to the high strength of the materials being tested, the desired energy stored in the incident bar is usually large, which makes it difficult to apply the pressure of the clamp. A large amount of pressure can lead to the plastic deformation of the bar, and low pressure can cause the bar slippage. Both of the issues represent failures of the experiments. Therefore, the success rate of this method is not high. In addition, the experimental loading process of this method is complicated, as it consumes time, elongates the experimental cycle, and decreases efficiency.

2.2. Explosive Loading TSHB. In 1971, Duffy et al. [40] originally used explosive loading to initiate a torsional wave. Explosive loading produced a torsional wave with short duration and short rising time. As shown in Figure 6, the torsional wave was generated by simultaneously detonating the two small charges of explosives, which were set symmetrically at the loading end of the incident bar. To obtain a pure torsional wave without a bending wave, equal impulses must be provided by each explosive charge. Thus, the charge of explosives must be the same and must be detonated simultaneously. Two explosives were connected in parallel to a common fuse, and tests shown that the time difference of the explosive charges is less than $0.1 \mu \mathrm{s}$. The advantage of explosive loading is the short rising time of the torsional wave compared to the prestored energy loading. A typical rising time is only 7 10 $\mu \mathrm{s}$, compared with $20 \sim 50 \mu \mathrm{s}$ for prestored energy loading. Using explosive loading TSHB, Duffy et al. [40] conducted high strain rate shear experiments on 1100-O aluminum, and the strain rate of the specimen reached up to $800 \mathrm{~s}^{-1}$. Subsequently, many scholars used explosive loading TSHB to conduct experiments on different materials, such as experiments on 1100-O aluminum conducted by Frantz and Duffy [49] and experiments on 1100-0 aluminum, OFHC copper, AZ31 B magnesium and commercially pure zinc conducted by Senseny et al. [50].

Although the advantage of explosive loading on the rising time of the torsional wave is obvious, some disadvantages are critical, which significantly hampered the wide usage of this technique. Due to the influence of unpredictable factors such as composition, charge, and dampness of the explosive, precisely controlling the shape of the torsional wave is difficult to achieve, which leads to poor repeatability of experiments. In addition, although the stress wave generated is almost entirely torsional, some low amplitude axial and bending waves are usually generated, which cannot be completely eliminated.

2.3. Direct Impact Loading TSHB. In 2011, the direct impact loading TSHB, which generated a torsional wave through the direct impact of a striker, was proposed by Nie et al. [41]. As shown in Figure 7, an impact pin was inserted in one end of the incident bar. The striker impacted the lower part of the impact pin at a certain velocity, and then, an instantaneous bending moment was generated at the end of the incident bar. Due to the asymmetric impact, the bending moment simultaneously generated a torsional wave and a bending wave in the bar. To eliminate the bending wave, a steel cushion was set at the right side of the bar to prevent any lateral displacement. Notably, this bending wave elimination method is effective when the expected amplitude of the torsional wave is low; however, if the expected amplitude of the torsional wave is high, the bending wave cannot be completely flittered out. Claus et al. [51] modified the direct 


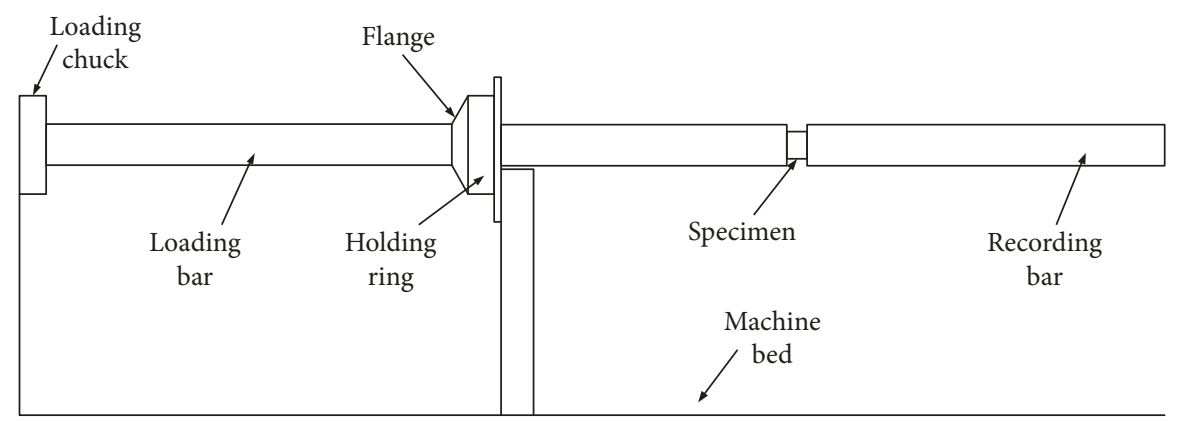

FIgURE 4: TSHB design by Lewis and Campbell [45].

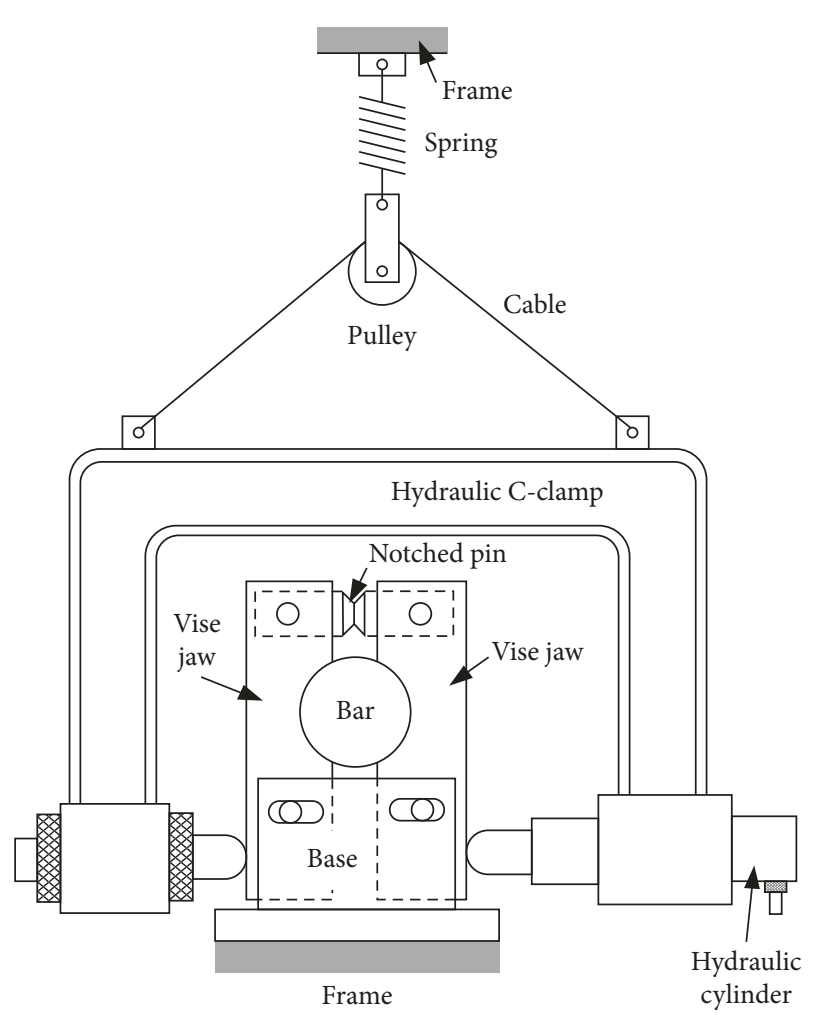

FIgURe 5: The clamp with a notch pin modified by Hartley et al. [48].

impact loading TSHB, as shown in Figure 8. They used SHPB to replace the striker. This modification enabled the pulse shaping technique to be used to profile the desired shape of the torsional wave. Typical considerations involve striker length, striker velocity, and pulse shaper geometry, which were used to generate the desired waveform in the SHPB, were directly applied to the direct impact loading TSHB to control the pulse length, amplitude, and rising time of the torsional wave, respectively. Compared with the prestored energy and explosive loadings, the direct impact loading improves the controllability of the torsional wave, saves experimental time, and raises the success rate.

However, there are also some shortcomings of the direct impact loading technique. In fact, this loading method can be explained as generating a torsional wave from the lateral pressure exerted on the bar end (for TSHB experiments, the lateral force or torque are characterized by very short durations, which are generally expressed as compressional

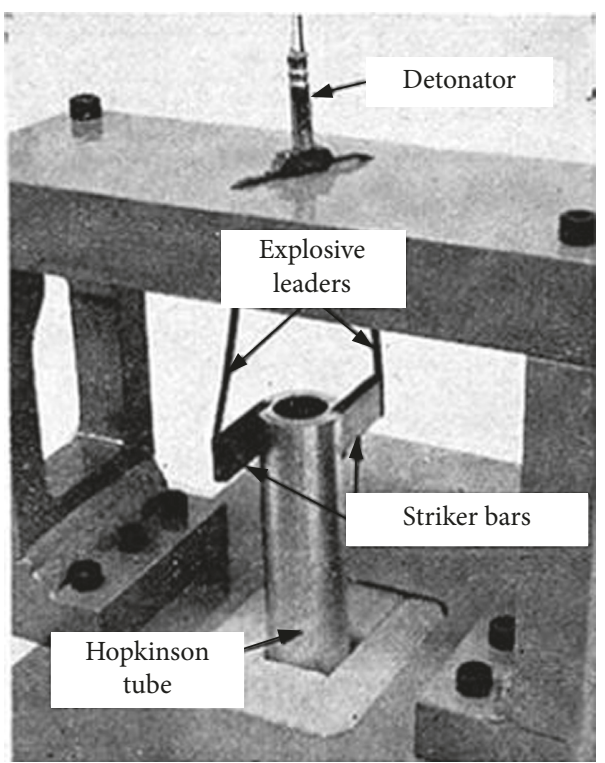

FIgURE 6: Explosive loading TSHB designed by Duffy et al. [40].

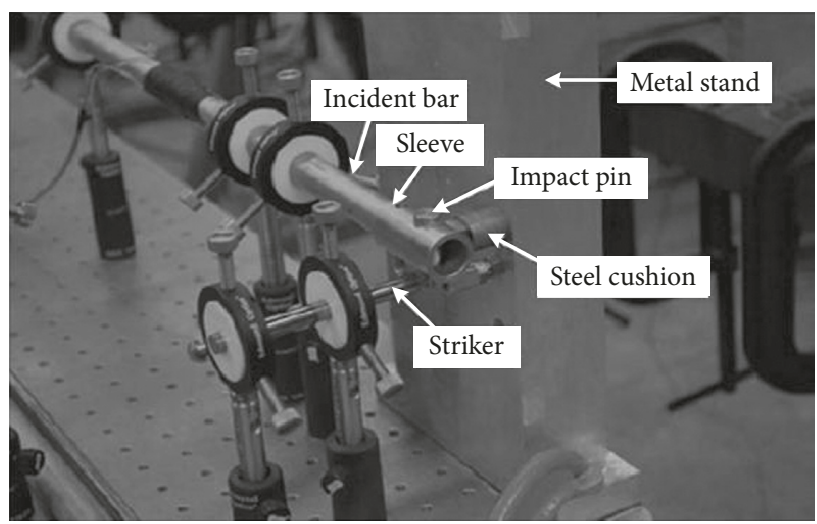

Figure 7: Direct impact loading TSHB designed by Nie et al. [41].

wave or torsional wave). Lateral forces generate bending moments [52], which are usually expressed as a bending wave while generating torque in the bar. Thus, the bending wave interference associated with this loading method is inevitable, and the larger the torsional amplitude required, the greater the bending wave interference and the more difficultly eliminating the bending wave. If the bending wave 


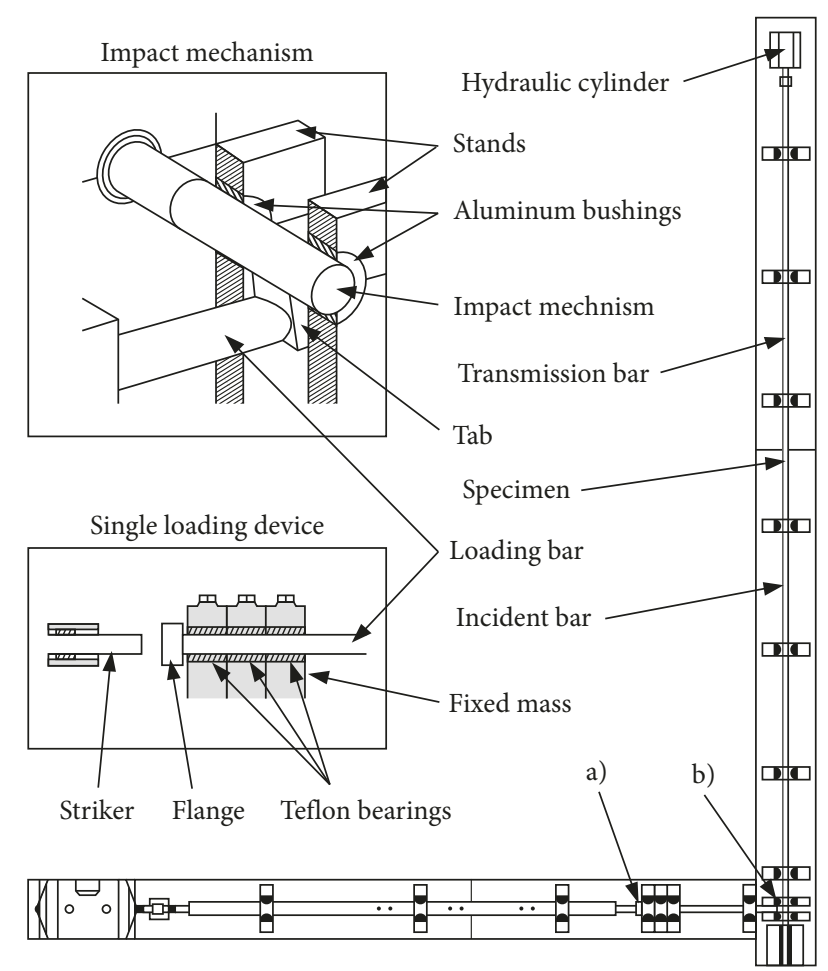

FIGURE 8: Direct impact loading TSHB developed by Claus et al. [51].

is not completely filtered out, the pure shear assumption of the specimens will be invalid. In addition, the existence of bending waves will seriously interfere with the torsional wave record, which will subsequently cause signal analyzing errors. In addition, if the test material has a great aggregate, such as concrete and rock, a larger incident bar is needed. Because this technique involve loading to one side of the bar end, there will be severe uniform stress on the cross section when the diameter of the bar is large. Therefore, the direct impact loading TSHB is not suitable for testing materials with very high strength or materials with a great amount of aggregates.

\subsection{Flywheel Loading TSHB and Electromagnetic Loading} TSHB. In recent years, to develop the TSHB for investigating more engineering materials, two new loading techniques, i.e., the flywheel loading and electromagnetic loading were provided by team of Fang et al. [42, 43] from Army Engineering University of PLA.

In 2016, Fang et al. [42] presented a flywheel loading TSHB, which is shown in Figure 9. The apparatus contains a motor, two electromagnetic clutches, a flywheel, an incident bar, a transmission bar, and a specimen. The magnetic force generated by the electromagnetic clutches can switch the position of the flywheel between the clutches. The steps in the experiment are as follows. First, the left electromagnetic clutch is turned on, which attracts and contacts tightly with the flywheel. Second, the motor is turned on, which drives the flywheel rotation at a certain speed. The kinetic energy is stored in the flywheel. Third, the clutch switch is switched over, and the flywheel disengages from the left clutch and connects suddenly to the right clutch. The friction between the flywheel and right clutch generates a torsional wave at the end of the incident bar.

The torsional wave generated by this apparatus has the following profile. The rising time is approximately $200 \mathrm{~ms}$, and the duration is approximately $600 \mathrm{~ms}$. The rising time of this torsional wave is too large compared with that generated by the aforementioned TSHB. However, it can be applied to the material tests at low to medium strain rates $\left(10^{-3} \sim 1 \mathrm{~s}^{-1}\right)$, which is still a blank for pure shear experiments.

In 2018, Fang et al. [43] invented an electromagnetic loading TSHB. As shown in Figure 10, the new type of TSHB was driven by an electromagnetic motor, which contains two electrical circuits: a stator circuit and a rotor circuit. The stator circuit, which contains the stator, electric source, and a switch can build a strong and constant magnetic field with a high strength constant current, which is approximately $5000 \mathrm{~A}$. The rotor circuit contains the rotor, electric source, and a switch. Under the magnetic field, the connection of the rotor circuit generates an instantaneous torque on the rotor. The torque is then transmitted into the incident bar as the torsional wave. The rising time and duration of the generated torsional pulse are $350 \mu \mathrm{s}$ and $2 \mathrm{~ms}$, respectively. The magnitude of the stress wave could be controlled by adjusting the currents of the rotor and stator circuits. The torsional wave of this feature can be applied to mechanical experiments in which the material is placed under medium to high strain rates $\left(10 \sim 10^{2} \mathrm{~s}^{-1}\right)$.

The newly designed TSHB can avoid the intrinsic problems of existing devices, i.e., the low success rate of the prestressed TSHB, poor pulse control of the blast loading TSHB, and the flexural wave interference of the direct impact loading TSHB. These techniques may be further implemented to expand testing to more materials, such as concrete and rock.

\section{General Description of the TSHB}

3.1. Components of a TSHB. There is no universal standard design for a TSHB; however, most TSHB share five common elements:

(1) Two long bars with a uniform cross section, i.e., the incident bar and transmission bar. In fact, there is no geometrical dispersion [33] of the torsional waves propagating in the elastic cylindrical bar, and solid or hollow bar shapes are both applicable to the TSHB. Generally, to match the hollow shape of the specimen and to magnify the amplitude of the recorded pulse signal, hollow bars (or tubes) are normally used in the TSHB. The bar ends should be machined orthogonally to the bar axis with a high level of accuracy (no standard tolerance available) to ensure good contact between the specimen and bars.

(2) A torsional stress wave loading system, which is capable of generating the desired torsional wave in the incident bar, which is described in Section 2. 


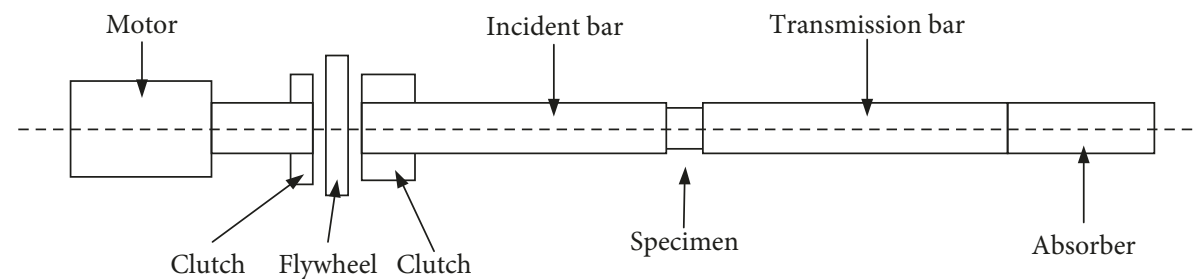

Figure 9: Sketch of the flywheel loading TSHB [42].

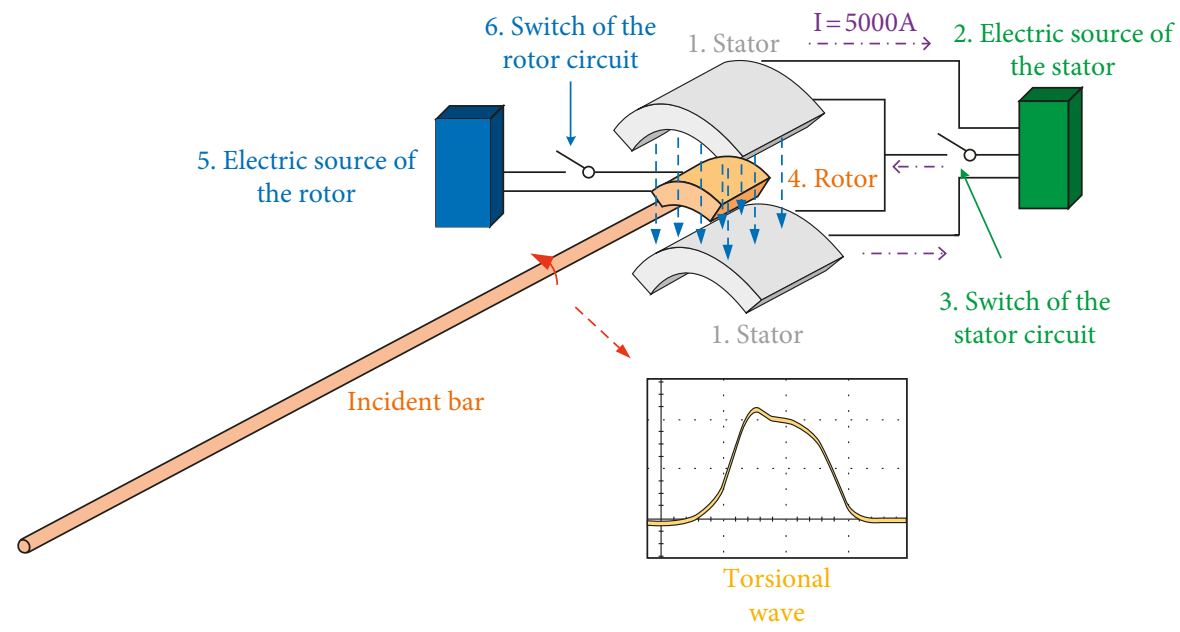

Figure 10: The electromagnetic loading TSHB [43].

(3) A bearing and alignment fixture for correct alignment. The incident bar, specimen, and transmission bar should be adjusted coaxially to maintain $1 \mathrm{D}$ wave propagation conditions.

(4) Strain gauges mounted on both bars to measure the stress wave histories in the bars, the direction of the strain rosette of the strain gauge is $45^{\circ}$ to the axial direction of the bars. Every position of the four strain gauges pasted to the bars is measured, which accesses the Wheatstone bridge through the whole bridge method to eliminate the interference of compressive and bending waves.

(5) The Wheatstone bridge, strain amplifier, and oscilloscope are necessary to transfer, magnify, and record the strain histories of the bars. A data analyzing system is needed to analyze the test results.

3.2. Elementary Assumptions. As a prerequisite to ensure the correctness of the experimental results, experiments conducted by TSHB should meet four elementary assumptions:

(1) One-dimensional stress wave propagation in the bars. The isotropic homogenous materials, such as steel and aluminum, are usually used to manufacture the bars. The bars should be long straight bars with a length to diameter ratio $\left(L_{\mathrm{B}} / D_{\mathrm{B}}\right)$ greater than 20 . The cross section of the bars in the axial direction is uniform, and the bars must remain in the elastic zone during the experiment.
(2) The stress equilibrium of the specimen, which could be expressed as $T_{\mathrm{I}}(t)+T_{\mathrm{R}}(t)=T_{\mathrm{T}}(t) . T_{\mathrm{I}}(t)$ is the incident wave, $T_{\mathrm{R}}(t)$ is the reflected wave, and $T_{\mathrm{T}}(t)$ is the transmitted wave. The stress equilibrium of the specimen is controlled by three factors, i.e., the length of the specimen, the rising time of the incident wave, and the specimen to the bar material mechanical impedance ratio. Both a shorter specimen and longer rising time of the incident wave can facilitate the stress equilibrium of the specimen. The influence of the mechanical impedance ratio is quite complicated, which is discussed in [53-56].

(3) The thin-walled tube structure of the specimen. Under torque, only the thin-walled tube, the thickness to average radius ratio $\left(t_{s} / r_{\mathrm{s}}\right)$ of which is smaller than 0.1 [52], can ensure the cross section of the specimen is approximately under the uniform pure shear.

(4) Full contact between the specimen and the bars. The surfaces of the specimen and the ends of the bars must reach a certain degree of flatness. The connection between the specimen and bar is also very important. Because there is no axial displacement in the TSHB experiments, as soon as the specimen and the bar separate, the torsional wave cannot be transmitted through. Therefore, an appropriate connection method should be selected to prevent any damage or breakage of the interface between the specimen and the bars. 
3.3. Traditional Stress Wave Analysis of TSHB. Experimental data acquired from the TSHB experiment mainly contains three waves, i.e., incident wave, reflected wave, and transmitted wave. A typical set of stress waves are shown in Figure 11, which are recorded by the oscilloscope as voltage signals $U(t)$. The strain wave, which is $\gamma(t)$, of the bars could be obtained by multiplying the voltage signal to the conversion factor $F$ :

$$
\gamma(t)=F \cdot U(t) .
$$

According to Equation (1), the incident strain wave $\gamma_{\mathrm{I}}(t)$, reflected strain wave $\gamma_{\mathrm{R}}(t)$, and transmitted wave $\gamma_{\mathrm{T}}(t)$ were obtained.

Figure 12 shows a sketch of the specimen in a typical TSHB experiment, the average shear strain rate history of the specimen can be obtained from the difference in the angular velocity between its two ends, which can be expressed as follows:

$$
\dot{\gamma}_{\mathrm{S}}(t)=\frac{r_{\mathrm{S}}}{L_{\mathrm{S}}}\left[\dot{\theta}_{1}(t)-\dot{\theta}_{2}(t)\right]
$$

where $\dot{\theta}_{1}(t)$ and $\dot{\theta}_{2}(t)$ are the angular velocities of the specimen ends where it attaches to the incident bar and transmission bar, respectively. $L_{\mathrm{S}}$ and $r_{\mathrm{S}}$ are the length and mean radius of the specimen, respectively.

According to the stress equilibrium of both ends of the specimen, the angular velocity difference can be calculated by the reflected stress wave.

$$
\dot{\theta}_{1}(t)-\dot{\theta}_{2}(t)=\frac{2\left[-T_{\mathrm{R}}(t)\right]}{J_{\mathrm{B}} \rho_{\mathrm{B}} C_{\mathrm{B}}},
$$

where $J_{\mathrm{B}}, \rho_{\mathrm{B}}$, and $C_{\mathrm{B}}$ are the polar moment of inertia, density, and torsional wave velocity of the specimen, respectively. $T_{\mathrm{R}}(t)$ is the reflected wave, which can be calculated as follows:

$$
T_{\mathrm{R}}(t)=\frac{G_{\mathrm{B}} J_{\mathrm{B}}}{r_{\mathrm{B}}} \gamma_{\mathrm{R}}(t)
$$

Substituting Equations (3) and (4) into Equation (2), the average strain rate history of the specimen is obtained as follows:

$$
\dot{\gamma}_{\mathrm{S}}(t)=\frac{2 C_{\mathrm{B}} r_{\mathrm{S}}}{r_{\mathrm{B}} L_{\mathrm{S}}}\left[-\gamma_{\mathrm{R}}(t)\right]
$$

Integrating the strain rate history, the average shear strain of the specimen is obtained as follows:

$$
\gamma_{\mathrm{S}}(t)=\int_{0}^{t} \dot{\gamma}_{\mathrm{S}}(t) d t
$$

The average shear stress of the specimen can be calculated from the torque it is under, which is denoted as $T_{\mathrm{S}}(t)$. According to the stress equilibrium of the interface between the specimen and transmission bar, the torque of specimen $T_{\mathrm{S}}(t)$ is equal to the torque of the transmission bar $T_{\mathrm{T}}(t)$. Therefore, the average shear stress of the specimen is expressed as follows:

$$
\tau_{\mathrm{S}}(t)=\frac{T_{\mathrm{T}}(t)}{2 \pi r_{\mathrm{S}}^{2} t_{\mathrm{S}}},
$$

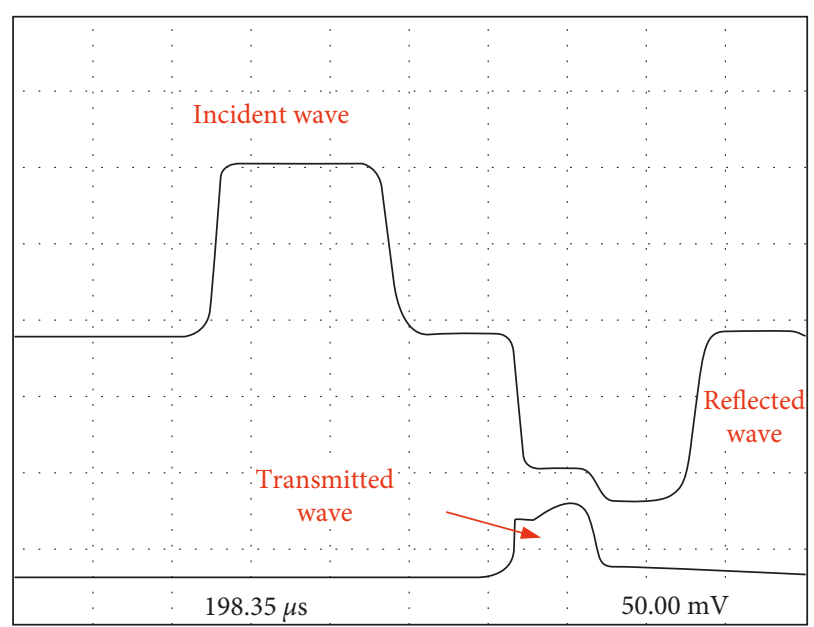

FIGURE 11: Typical three wave signals of the TSHB experiment [57].

where $t_{\mathrm{S}}$ is the wall thickness of the tubular specimen. $T_{\mathrm{T}}(t)$ can be calculated by the following:

$$
T_{\mathrm{T}}(t)=\frac{G_{\mathrm{B}} J_{\mathrm{B}}}{r_{\mathrm{B}}} \gamma_{\mathrm{T}}(t) .
$$

Substituting Equation (8) into Equation (7), the average shear stress of the specimen is obtained by the following:

$$
\tau_{\mathrm{S}}(t)=\frac{G_{\mathrm{B}} J_{\mathrm{B}}}{2 \pi r_{\mathrm{S}}^{2} t_{\mathrm{S}} r_{\mathrm{B}}} \gamma_{\mathrm{T}}(t) .
$$

Therefore, the stress-strain curves can be obtained by combining the results calculated by Equations (6) and (9).

3.4. Pulse Shaping Technique. To facilitate the stress equilibrium and reach a constant strain rate of the specimen, the pulse shaping technique is usually used in Hopkinson bar experiments [58]. A pulse shaper, which is made of different materials such as rubber and copper, is placed on the path of the propagating stress wave to filter out the high frequency waves and elongate the rising time of the stress wave. In 1975, Eleiche and Duffy [59] provided a pulse shaping technique to eliminate the undesired bending wave generated by the explosive loading TSHB. Thereafter, Gilat [57] reported this technique in detail in his research. Figure 13 shows a sketch of TSHB with the pulse shaping technique. The incident bar is cut off in the middle, and a pulse shaper is bonded between the two parts. A photo of the pulse shaper is shown in Figure 14, which is divided into upper and lower parts. The upper part serves to filter out the bending waves and the lower part filters out the high frequency torsional waves. When the stress wave propagates through the pulse shaper, only the desired smooth, low-frequency torsional wave remains, which further propagates in the incident bar and finally loaded on the specimen.

The direct impact loading TSHB [51] is more flexible in shaping the torsional wave. The mature SHPB pulse shaping technique can be directly used. A pulse shaper is attached to the end of the incident bar. Under impact of the striker, the pulse shaper is plastically deformed, which filters the high 


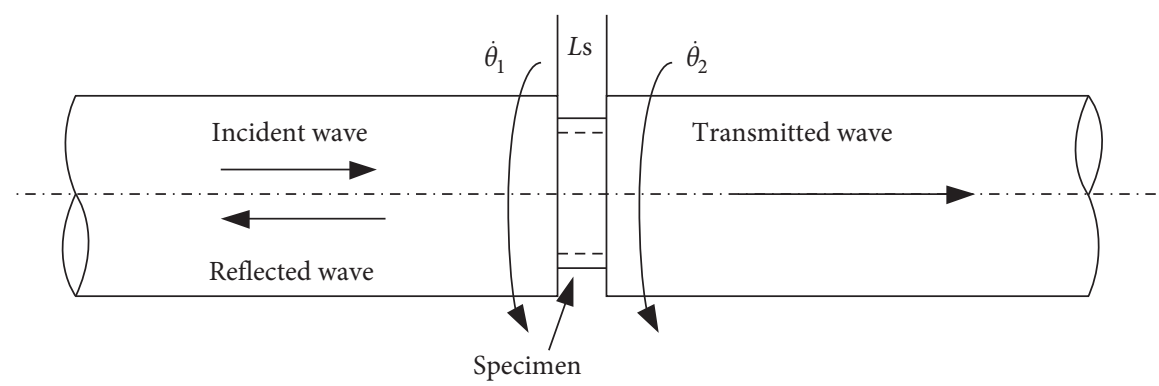

FIgURE 12: Sketch of the specimen in the TSHB experiment [57].

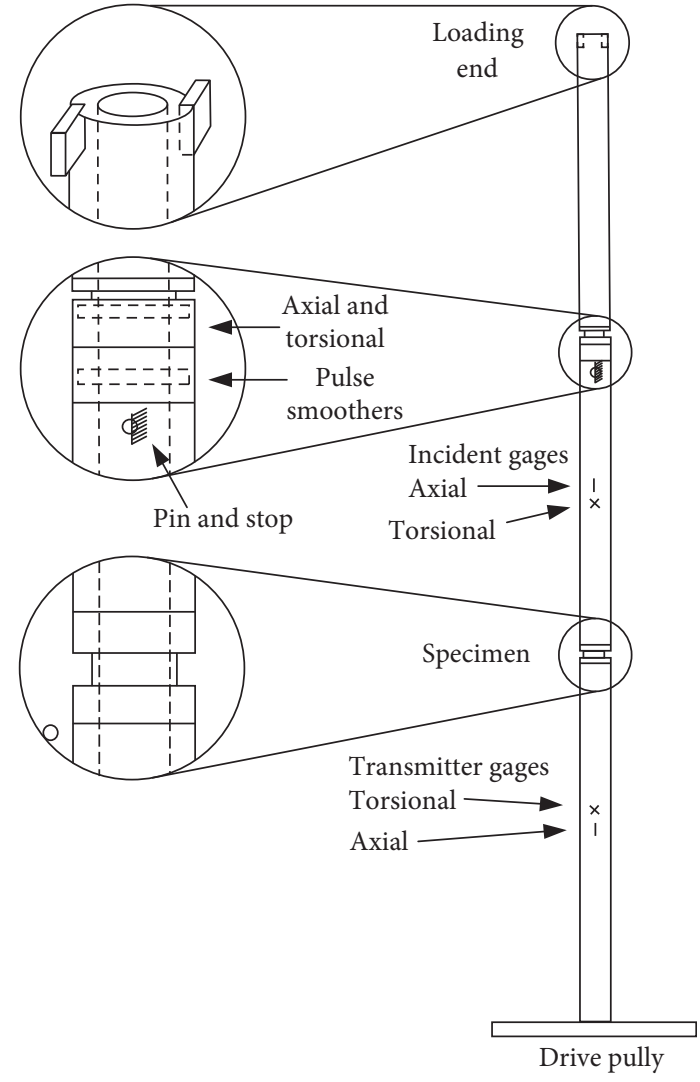

FIGURE 13: Sketch of the pulse shaping technique for TSHB [57].

frequency portions of the stress wave and elongates the rising time of the stress wave. In addition, the pulse duration of the torsional wave can be adjusted by changing the striker length, and the amplitude of the torsional wave can be controlled by varying the velocity of the striker.

3.5. Specimen Design. A specimen in the TSHB experiment usually consists of two parts, which are shown in Figure 15. One part is the thin-walled test portion, and the other part is the flanges at both sides of the test portion.

Three principles should be followed when designing the thin-walled test portion of the specimen.

(1) The thin-walled tube structure must be satisfied, that is, the wall thickness to the average radius ratio $\left(t_{s} / r_{s}\right)$ should be smaller than 0.1 .

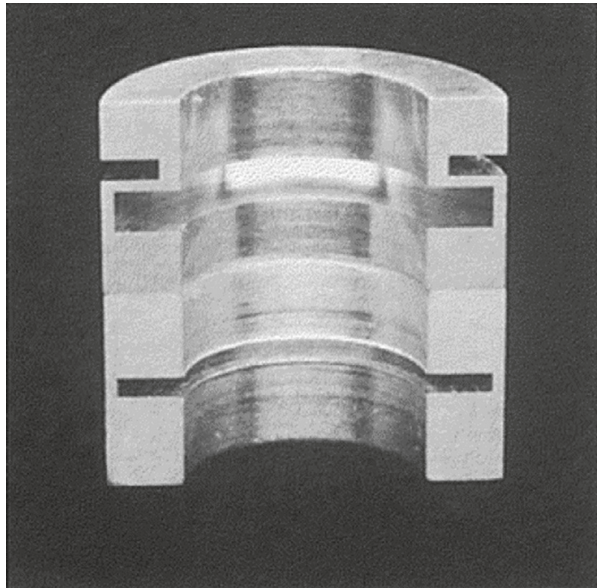

FIgUre 14: The pulse shaper for TSHB [57].

(2) The length of the specimen should be short to allow the stress equilibrium assumption to be satisfied.

(3) To ensure the uniformity of the specimen, the minimum dimension in every direction of the test portion should be larger than 10 times the maximum grain size of the material.

The flanges are mainly responsible for the connection between the specimen and bars to transmit the torsional stress wave. The flanges are usually designed as a thickwalled tube. The inner diameter is the same as the test portion. Different outer shapes are machined for different connecting mechanism, which is shown in Figure 15. The cylindrical flange, which has outer diameter that is the same as the bars, is machined for cementing to the bar ends with epoxy cement, as shown in Figure 15(a). The cylindrical flange has the advantage of being easily machined and is commonly used for materials with low strengths, such as 1100-O aluminum [60] and OFHC copper [38], in which the cement can provide enough strength to ensure that the epoxy cement layers do not break during the experiment. The hexagonal flange, which has outer shapes that are the same as the groove of bar ends, is riveted to the bar end, as shown in Figure 15(b). The hexagonal flange is usually used for materials with high strength, such as alloy steel.

3.6. Single Pulse Loading Technique. For TSHB experiments, the specimen is tightly connected to the incident and 


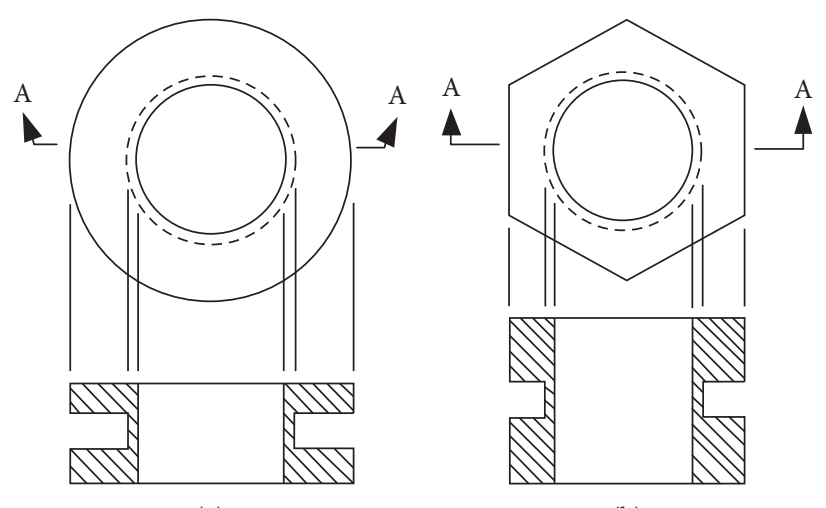

(a)

(b)

FIgURE 15: Two typical specimen types used in TSHB experiments. (a) Tubular specimen with cylindrical flanges. (b) Tubular specimen with hexagonal flanges [61].

transmission bars. After the incident wave loads on the specimen, the specimen is still connected to the incident and transmission bars. Therefore, the reflected wave reflected from the other end of the incident bar and the transmitted wave reflected from the other end of the transmission bar will repeatedly load on the specimen, which will result in secondary or even multiple loading. The repeated loading makes it impossible to observe the real failure morphology of the specimen under a single loading. In addition, the deformation or failure characteristic of the specimen is unable to relate to the stress-strain curve. Also, there is no axial displacement during the TSHB experiment, and the mature single-pulse loading technique of SHPB [62-64] cannot be directly applied to the TSHB.

Xue et al. $[65,66]$ firstly developed a single-pulse loading technique for TSHB. The transmitted wave was completely transmitted out of the transmission bar by introducing an absorption bar and an actuator, but the reflection of the reflected wave in the incident bar was not solved. Thereafter, Xue et al. $[67,68]$ improved the single pulse loading technique, which allowed the reflected wave to transmit out, and a single loading for the entire TSHB apparatus was achieved. The sketch is shown in Figure 16, where the loading chuck was designed as a hollow, the loading end of the incident bar was changed to free from fixed, and both modifications allowed the incident bar to pass through the loading chuck. The absorption bar was set at both ends of the transmission bar and incident bar, and a unidirectional torque transmission actuator was used as the connector. The angular velocity induced by the torsional wave difference between the incident bar (or transmission bar) and absorption bars achieved an automatic separation.

\subsection{Combined Loading}

3.7.1. Combined Compression and Shear Experiment. Lewis and Goldsmith [69] developed the TSHB into a combined dynamic compression-torsion loading apparatus. As shown in Figure 17, the loading mechanisms of the compressional and torsional waves were set independently.
The prestored energy loading mechanism on the torsional wave was installed at the end of the incident bar, and the impact loading mechanism on the compressional wave was installed at the end of the transmission bar. A cross-trigger line was placed at the end of the transmission bar, which acted as a clamp-releasing switch for the prestored energy loading mechanism. Simultaneously, as the striker stroke the transmission bar, the clamp control loop was connected, which released the clamp to generate the torsional wave. The time difference between generating the compressional wave and torsional wave was ensured within a few microseconds. However, this independent loading method of the compression and torsional waves renders uncertainty in the time difference. To conduct dynamic friction experiments on materials, Espinosa et al. [70] modified the prestored energy loading TSHB and achieved a dynamic compression-torsion combined loading. The apparatus is depicted in Figure 18. Different from the combined loading method proposed by Lewis and Goldsmith [69], the compressional wave and torsional wave loading mechanisms of the modified TSHB were installed on the same side of the incident bar. The function of the clamp was extended, which can not only prevent the incident bar from being twisted, but also from being compressed. The prestored compressional and torsional energies were released by the common clamp, which can generate the compressional wave and torsional wave simultaneously. The issue of the time difference on generating the compressional wave and torsional wave was resolved. Notably, although the compressional wave and the torsional wave are generated at the same moment, their wave velocities are different. They reach the specimen, therefore, at different moments after propagating through the incident bar, and the time difference is a certain value. Espinosa et al. [70] provided a detailed discussion of this time difference. Using this apparatus, Espinosa et al. [70] conducted a dynamic friction experiment, where the specimen differed from those of the normal TSHB experiments. Two friction pairs were employed: a U-shaped friction pair was glued to the end of the incident bar, and a disk-shaped friction pair was glued to the end of the transmission bar, which is shown in Figure 19. Based on the apparatus proposed by 


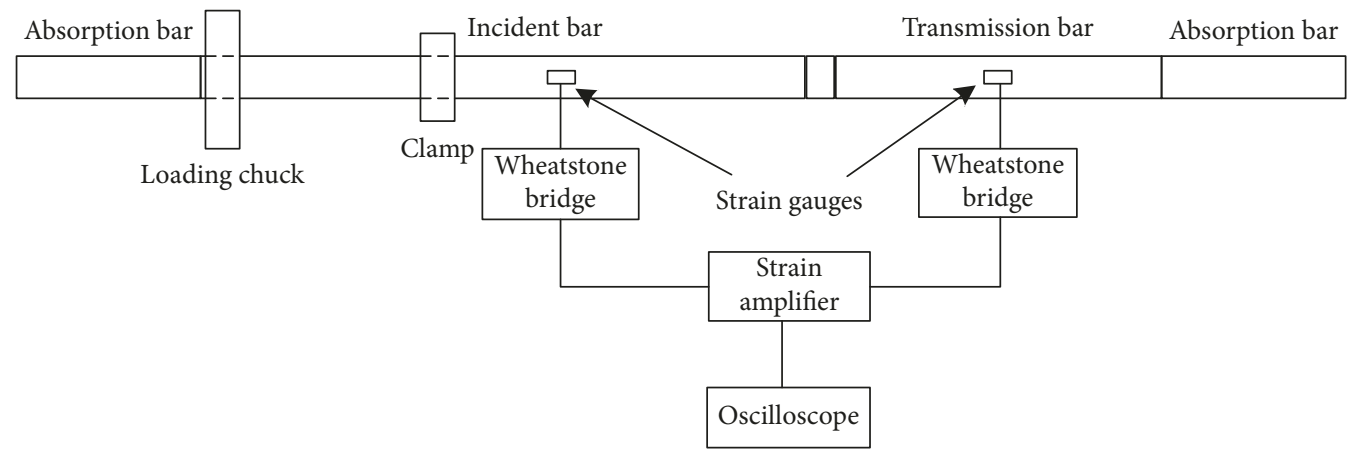

FIgURE 16: Schematic diagram of the single pulse loading TSHB [68].

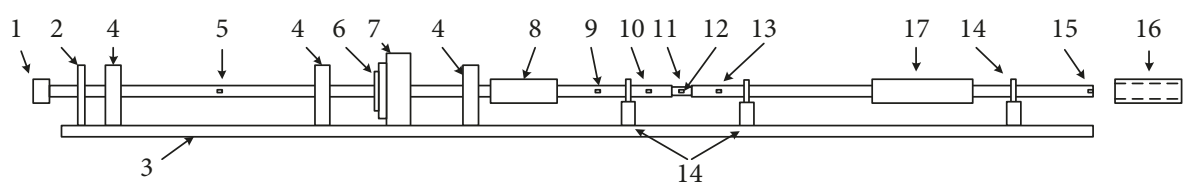

FIGURE 17: A biaxial TSHB for simultaneous torsion and compression designed by Lewis and Goldsmith [69]. 1: torque handle; 2: clamping block; 3: machine bed plate; 4: bearing block; 5: static torque gauge; 6: holding flange; 7: holding frame; 8: torsional bending suppressor; 9: bending strain gauge; 10: longitudinal strain gauge; 11: specimen; 12: specimen strain gauge; 13: torsion strain gauge; 14: alignment post; 15: crossed wire trigger; 16: air gun; and 17: longitudinal bending suppressor.

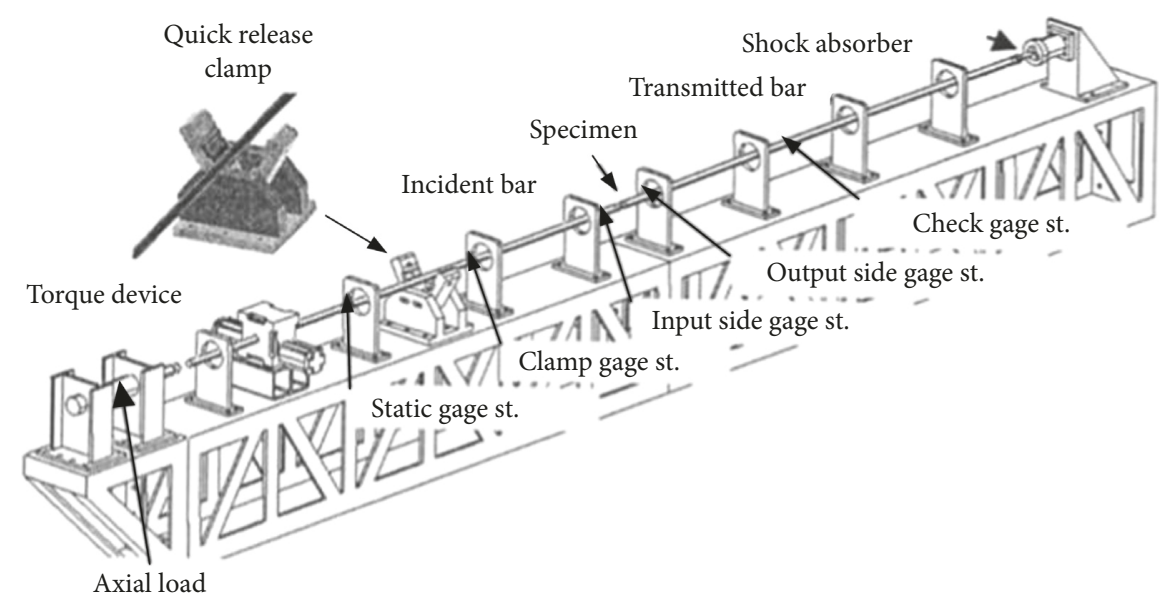

FIGURE 18: TSHB for dynamic compression-torsion modified by Espinosa et al. [70].

Espinosa et al. [70], Huang and Feng [71] made further modifications, which realized the single pulse loading of the dynamic compression-torsion.

Different from the dynamic compression-torsion proposed by Espinosa et al. [70], the friction experiment on the material was conducted by Rajagopalan et al. [72-74] through the combined loading of static compression and dynamic torsion. The apparatus was improved from the prestored energy loading TSHB, which is shown in Figure 20. An axial pressure mechanism, which was used to apply static axial pressure on the specimen, was installed on the end of the incident bar. For the friction pairs in the experiment, one pair was designed with a thin-walled shape and glued to the end of the incident bar. The other part was substituted by a rigid support. During the experiment, static pressure was firstly applied to the end of the incident bar by the axial pressure loading mechanism. When the pressure reached the predetermined value, the clamp was tightened to fix the incident bar, and then, the torsion head turned to twist the incident bar and stored torsional energy in the bar. The clamp was released when the stored torsional energy reached the predetermined value, and then, the specimen, which was already under axial static compression, was loaded by the torsional wave.

3.7.2. Combined Loading Tests for Quasistatic and High Strain Rate Torsion. In 1972, Frantz and Duffy [49] improved the explosive loading TSHB, which enabled the device to perform a combined quasistatic torsion and high strain rate torsion experiment. The mechanism for loading the torsional wave was set at the end of the incident bar, and 

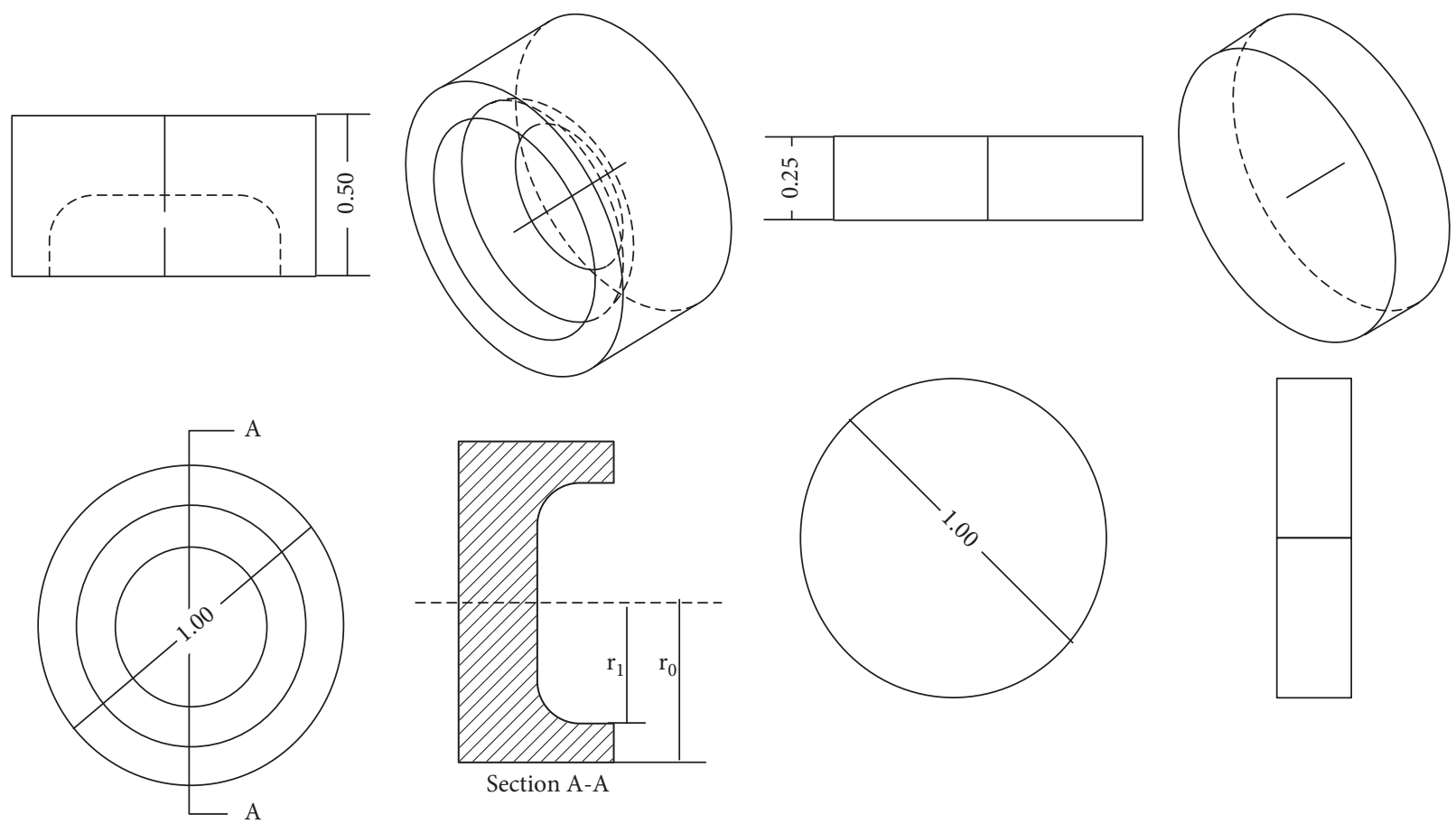

(a)

(b)

FIGURE 19: Friction specimen designed by Espinosa et al. [70]: (a) u-shaped disk attached to the incident bar and (b) disk attached to the transmission bar.

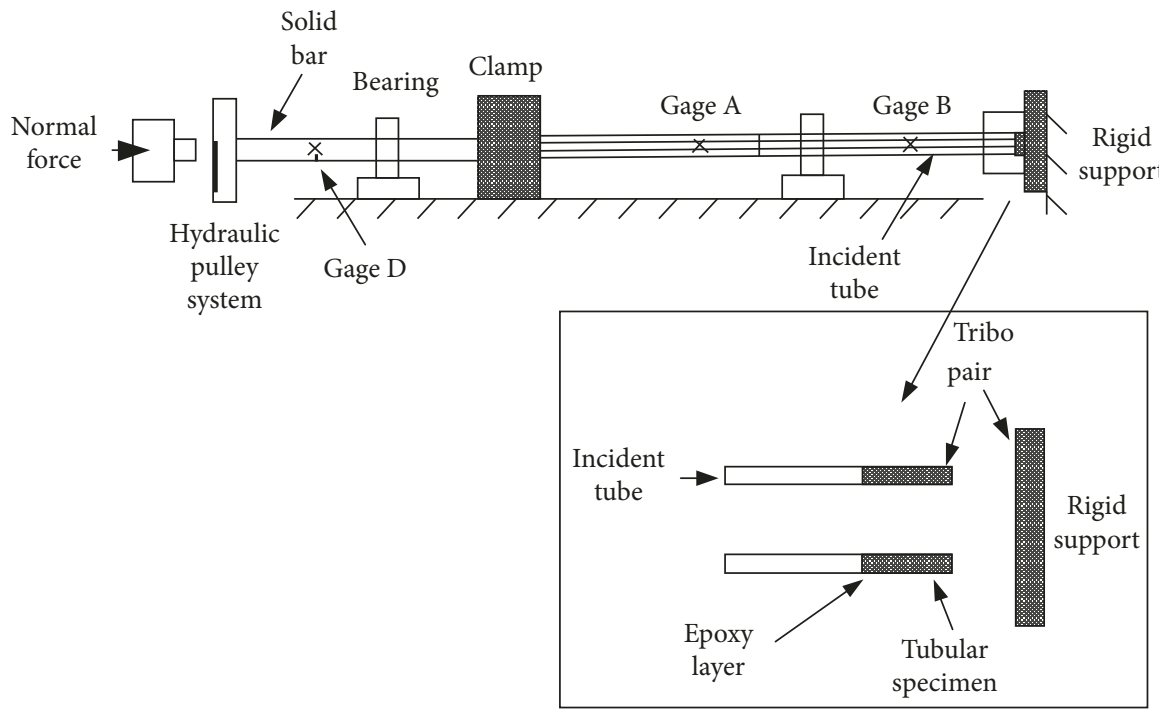

Figure 20: TSHB for static compression and dynamic torsion developed by Rajagopalan and Prakash [72].

the mechanism for loading the quasistatic torque was placed at the end of the transmission bar. Therefore, the specimen was loaded at the double ends, the end connect to the transmission bar provided a quasistatic torque, and the end connect to the incident bar provided a dynamic torsion load. Frantz and Duffy [49] used this apparatus in the incremental strain rate experiment. The strain rate of the aluminum specimen increased from $5 \times 10^{-5} \mathrm{~s}^{-1}$ to $850 \mathrm{~s}^{-1}$ in $10 \mathrm{~ms}$. Campbell et al. [75] used the same apparatus to study the influence of the strain rate and strain rate history on the strength of materials. Similar to the explosive loading TSHB, the prestored energy loading TSHB have also been developed for the combined loading of quasistatic and high strain rate torsions [57], as shown in Figure 21. It has been applied to test the strain rate effects on incremental torsional shear [76] and reverse torsional shear [21] of materials.

3.8. Temperature Experiment. The temperature experiment on a SHPB (high-temperature experiment is given as an 


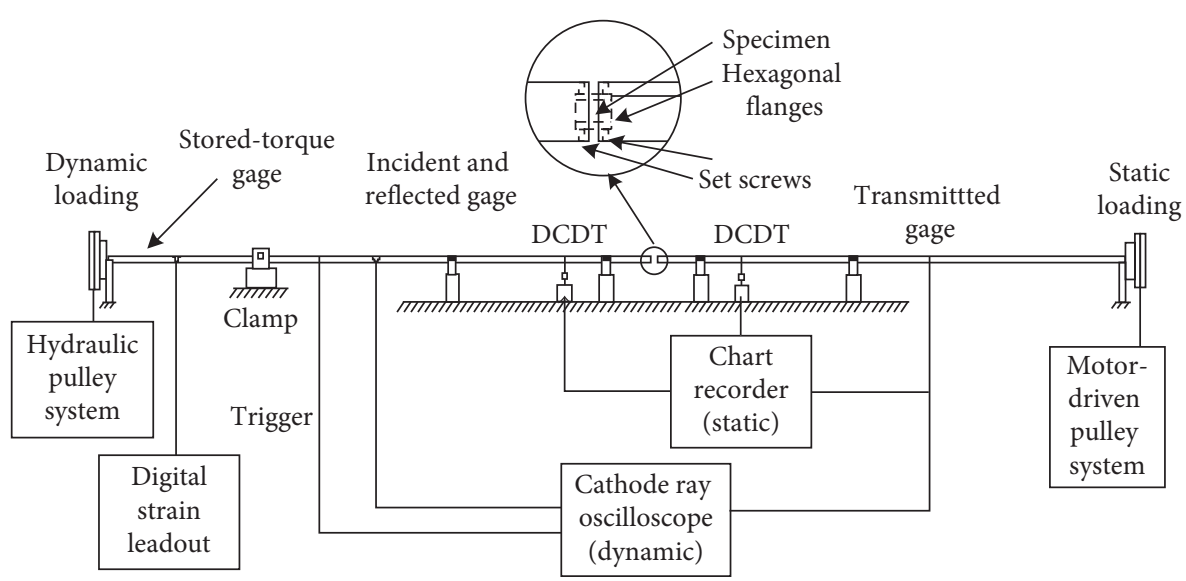

FIGURE 21: Schematic of a prestored energy TSHB modified for incremental strain rate experiments [57].

example; low temperature experiment is similar) was conducted earlier than the temperature experiment on a TSHB. The experimental method for a SHPB can be divided into two types: (1) a method which involves simultaneously heating the specimen and bar ends and the temperature effect correction in the bars is necessary [77, 78]; (2) a method which involves heating the specimen individually and the temperature effect correction in the bars is unnecessary [79-81]. In the first method, the specimen is first placed at the predetermined position in the hightemperature furnace. Then, the ends of the incident bar and transmission bar are inserted into the furnace and connected to the specimen. Subsequently, the specimen and parts of the bars that remained in the furnace are heated. When the temperature of the specimen reaches the predetermined value, the stress wave is then loaded. The influence of the temperature gradient on the stress wave propagation in the bar must be corrected when processing the experimental data. In the second method, only the specimen is heated in the high-temperature furnace. When the temperature reaches the predetermined value, the specimen is rapidly installed through the chute. A laser sensor triggers the cylinder to push the bars to connect to the specimen, and the striker is then launched to generate the stress wave. The experimental process exhibits an automation design, which ensures that the time from opening the furnace door to loading the stress wave is less than $2 \mathrm{~s}$ to avoid excessive heat loss of the specimen.

In contrast to the propagation of the compressional wave, the tight connection of the two surfaces is a premise for the torsional wave to transmit from one media to another. Thus, in the TSHB experiment, the specimen, incident bar, and transmission bar must be connected (bonding or riveting) tightly during the experiment. Therefore, the second method for a SHPB is not applicable to a TSHB, and the specimen must be assembled with the bars when heating for the TSHB. The heat transfer between the specimen, incident bar, and transmission bar are unavoidable, and the temperature gradients in the bars must inevitably occur. The temperature gradient affects the wave impedance of the bar material; therefore, the shape of the pulse will deform when propagating through the bars, which causes significant difficulties for extracting the stress-strain curve of the specimen by the recorded pulses.

In 1963, Chiddister and Malvern [77] proposed the temperature effect correction method for SHPB; this method is also suitable for TSHB. Although the calculation is accurate, the derivation process is tedious. Eleiche and Duffy [59] proposed a novel approach. The temperature effect on the bar wave impedance is offset by the gradually changing gradient of the bar's cross section. Therefore, the bar wave impedance remains constant for a specific temperature gradient, and the complex correction process for the collected waveforms is avoided. However, this method has certain limitations. A specific temperature gradient only corresponds to a bar with a specific cross section gradient. Due to influencing factors such as the external environment, precisely controlling the specific temperature gradient of the bar is difficult. In addition, since the temperature is the parameter to study, the bars with different cross section gradients need to be designed for different temperatures, which greatly raises the cost of the experiment. By modifying the connection method of the specimen and the bars and integrating a rapid heating device, Gilat and $\mathrm{Wu}$ [82] developed a TSHB that can rapidly heat a specimen while maintaining the bars at room temperature. As shown in Figure 22, the specimen did not directly contact with the bar ends, and the torque was transmitted through two specially designed adaptors. Each adaptor was fixed to the specimen by eight pins, which reduced the contact area while ensuring a uniform stress distribution on the specimen. The adaptors were made of titanium, which has extremely low thermal conductivity, and thus, heat could not easily propagate across the adaptor to the bars. The high-temperature of a specimen heated by this method can reach $650 \sim 1060^{\circ} \mathrm{C}$.

\section{Conclusions}

In this paper, the TSHB was reviewed according to the loading mechanisms, such as prestored energy loading, explosive loading, direct impact loading, flywheel loading, and electromagnetic loading. The main work of Baker and Yew [19], Duffy et al. [40], Nie et al. [41], and Fang et al. $[42,43]$ were reviewed in detail. A review of the design 


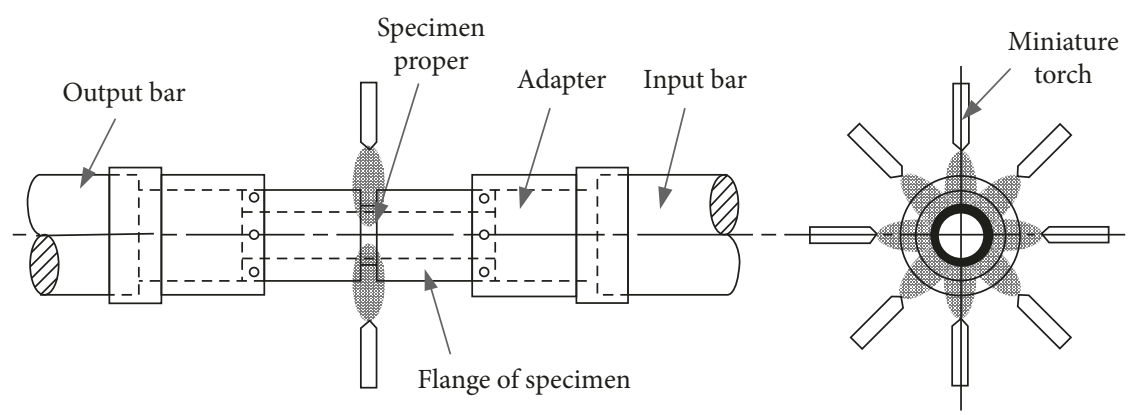

FIGURE 22: High-temperature technique for TSHB proposed by Gilat and Wu [82].

methods, data analysis, new technologies, and new methods of typical TSHB experiments were also provided.

As the main apparatus for testing the shear mechanical properties of materials with medium to high strain rates $\left(10^{1} \sim 10^{4} \mathrm{~s}^{-1}\right)$, the TSHB has been applied in certain ranges. Although a universal standard for TSHB has not been built, some basic assumptions in the experimental design need to be satisfied: assumptions of one-dimensional stress wave propagation, stress equilibrium of the specimen, and the thin-walled tube structure of the specimen $\left(t_{\mathrm{S}} / r_{\mathrm{S}} \leq 0.1\right)$.

To eliminate the bending wave interference generated during the loading of a torsional wave, the bending wave filtering technique was proposed. To shape the torsional wave for facilitating the stress equilibrium of the specimen, the torsional wave shaping technique was developed. To satisfy the experimental requirement for materials with different strengths, cylindrical flanges and hexagonal flanges for the specimen were, respectively, designed. The development of the single-pulse loading technique enabled the experimental stress-strain curve to correspond to the failure and damage characteristics of the specimen. The development of a temperature experiment enabled researchers to study the combined effects of temperature and strain rate on the mechanical behavior of materials. Combined loading methods, such as static compression-dynamic torsion, dynamic compression-dynamic torsion, static torsiondynamic torsion, and incremental strain rate torsion, enabled researchers to study mechanical properties of materials in complex stress conditions.

Considering the developing state of the TSHB, we believe that the following aspects deserve further study:

(1) Extending the range of applicable materials. The current TSHB is primarily employed to investigate materials with small and uniform aggregates, such as metal. However, due to technical bottlenecks, this experimental technique is unsuitable for other common materials, such as brittle materials (ceramic [83] and rocks [84]), low-wave-impedance soft materials (rubber [85], foam [86], and biomaterials [87]), and composite materials (concrete [88] and fiber composites [89]). Therefore, shear experimental data for high strain rates are lacking for a lot of materials. Developing existing techniques to expand the applicability range of TSHB shows practical significance.
(2) Developing the combined loading technique. After several decades of development, many of the aforementioned combined loading methods have been developed from TSHB. However, additional complex loading methods, such as tensile-torsion and tensile-compression-torsion, have not been reported. In daily life, materials are usually in complex stress states. Developing the combined loading technique is important for understanding the mechanical properties of materials, which can greatly benefit material modeling, numerical simulation, and engineering design.

\section{Conflicts of Interest}

The authors declare that there are no conflicts of interest regarding the publication of this paper.

\section{Acknowledgments}

The authors acknowledge the financial supports from the National Natural Science Foundation of China (grant nos. 51622812 and 51427807), National Basic Research Program of China (no. 2015CB058003) and China Postdoctoral Science Foundation (no. 2017M613379).

\section{References}

[1] Y. Cui, F. Lu, Y. Lin et al., "Review on dynamic shear techniques by Hopkinson bar," in Proceedings of 4th National Symposium on Explosion Mechanics Experimental Technology Conference of China, Hefei, China, 2006.

[2] D. R. Ireland, "Critical review of instrumented impact testing," in Proceedings of Welding Institute/ASM International Conference on Dynamic Fracture Toughness, vol. 1, pp. 47-62, Englewood Cliffs, NJ, USA, 1977.

[3] W. Chen and B. Song, Split Hopkinson (Kolsky) Bar: Design, Testing and Applications, Springer Science \& Business Media, Berlin, Germany, 2010.

[4] Z. Tang, "Progress and application of combined compression and shear wave loading technique," Advanced in Mechanics, vol. 37, no. 3, pp. 398-408, 2007, in Chinese.

[5] P. Johnson, B. Stein, and R. Davis, "Measurement of dynamic plastic flow properties under uniform stress," in Symposium on Dynamic Behavior of Materials, ASTM International, West Conshohocken, PA, USA, 1963.

[6] F. I. Niordson, "A unit for testing materials at high strain rates," Experimental Mechanics, vol. 5, no. 1, pp. 29-32, 1965. 
[7] W. P. Rogers and S. Nemat-Nasser, "Transformation plasticity at high strain rate in magnesia-partially-stabilized zirconia," Journal of the American Ceramic Society, vol. 73, no. 1, pp. 136-139, 1990.

[8] G. H. Staab and A. Gilat, "A direct-tension split Hopkinson bar for high strain-rate testing," Experimental Mechanics, vol. 31, no. 3, pp. 232-235, 1991.

[9] W. Li, H. Xie, and Q. Wang, "An experimental study for the dynamic split tension of marble disk using SHPB," Explosion and Shock Waves, vol. 26, no. 1, pp. 12-20, 2006, in Chinese.

[10] S. Hu, L. Zhang, H. Wu et al., "Experimental study on spalling strength of concrete," Engineering Mechanics, vol. 21, no. 4, pp. 128-132, 2004, in Chinese.

[11] C. Ruiz and R. A. W. Mines, "The Hopkinson pressure bar: an alternative to the instrumented pendulum for Charpy tests," International Journal of Fracture, vol. 29, no. 2, pp. 101-109, 1985.

[12] A. R. Dowling, J. Harding, and J. D. Campbell, "The dynamic punching of metals," Journal of the Institute of Metals, vol. 98, pp. 215-224, 1970.

[13] J. Harding and J. Huddart, "The use of the double-notch shear test in determining the mechanical properties of uranium at very high rates of strain," in Proceedings of 2 nd Conference on the Mechanical Properties of Materials at High Rates of Strain, pp. 49-61, Oxford, UK, March 1979.

[14] J. R. Klepaczko, "An experimental technique for shear testing at high and very high strain rates. The case of a mild steel," International Journal of Impact Engineering, vol. 15, no. 1, pp. 25-39, 1994.

[15] A. S. Abou-Sayed, R. J. Clifton, and L. Hermann, "The oblique-plate impact experiment," Experimental Mechanics, vol. 16, no. 4, pp. 127-132, 1976.

[16] R. J. Clifton and R. W. Klopp, "Pressure-shear plate impact testing," in ASM Handbook, Mechanical Testing and Evaluation, H. Kuhn and D. Medlin, Eds., vol. 8, pp. 457-461, ASM International, Russell, OH, USA, 2002.

[17] M. Okada, N. S. Liou, and V. Prakash, "Dynamic shearing resistance of molten metal films under high pressures and extremely high shearing rates," Experimental Mechanics, vol. 42, no. 2, pp. 161-171, 2002.

[18] K. S. Kim, R. J. Clifton, and P. A. Kumar, "Combined normal and transverse displacement interferometer with an application to impact of y-cut quartz," Journal of Applied Physics, vol. 48 , no. 10 , pp. 4132-4139, 1977.

[19] W. E. Baker and C. H. Yew, "Strain-rate effects in the propagation of torsional plastic waves," Journal of Applied Mechanics, vol. 33, no. 4, p. 917, 1966.

[20] T. Nicholas and J. E. Lawson, "On the determination of the mechanical properties of materials at high shear-strain rates," Journal of the Mechanics and Physics of Solids, vol. 20, no. 2, pp. 57-64, 1972.

[21] A. M. Eleiche and J. D. Campbell, "Strain-rate effects during reverse torsional shear," Experimental Mechanics, vol. 16, no. 8, pp. 281-290, 1976.

[22] G. Yang and Y. Song, "An experimental study on strain rate effect of L4 pure aluminum at very high rate of deformation," Acta Mechanica Solida Sinica, vol. 2, article 1302136, 1986 in Chinese.

[23] L. Tian, "Study on dynamic torsional test of Al-Li alloy," Explosion and Shock Waves, vol. 12, no. 1, pp. 68-76, 1992, in Chinese.

[24] A. Marchand and J. Duffy, "An experimental study of the formation process of adiabatic shear bands in a structural steel," Journal of the Mechanics and Physics of Solids, vol. 36, no. 3, pp. 251-283, 1988.

[25] G. T. Gray, "Classic split-Hopkinson pressure bar testing," in ASM handbook. Mechanical testing and evaluation, $\mathrm{H}$. Kuhn and D. Medlin, Eds., vol. 8, pp. 1027-1067, ASM International, Russell, OH, USA, 2000.

[26] B. A. Gama, S. L. Lopatnikov, and J. W Gillespie Jr., "Hopkinson bar experimental technique: a critical review," Applied Mechanics Reviews, vol. 57, no. 4, pp. 223-250, 2004.

[27] F. Jiang and K. S. Vecchio, "Hopkinson bar loaded fracture experimental technique: a critical review of dynamic fracture toughness tests," Applied Mechanics Reviews, vol. 62, article 060802, 2009.

[28] K. Xia and W. Yao, "Dynamic rock tests using split Hopkinson (Kolsky) bar system-a review," Journal of Rock Mechanics and Geotechnical Engineering, vol. 7, no. 1, pp. 27-59, 2015.

[29] A. Kidane, H. L. Gowtham, and N. K. Naik, "Strain rate effects in polymer matrix composites under shear loading: a critical review," Journal of Dynamic Behavior of Materials, vol. 3, no. 1, pp. 110-132, 2017.

[30] H. Kolsky, "An investigation of the mechanical properties of materials at very high rates of loading," Proceedings of the Physical Society, vol. 62, no. 11, pp. 676-700, 1949.

[31] E. D. H. Davies and S. C. Hunter, "The dynamic compression testing of solids by the method of the split Hopkinson pressure bar," Journal of the Mechanics and Physics of Solids, vol. 11, no. 3, pp. 155-179, 1963.

[32] J. M. Lifshitz and H. Leber, "Data processing in the split Hopkinson pressure bar tests," International Journal of Impact Engineering, vol. 15, no. 6, pp. 723-733, 1994.

[33] L. Wang, Foundation of Stress Waves, National Defense Industry Press, Beijing, China, 1985, in Chinese.

[34] T. Nicholas, "Strain-rate and strain-rate-history effects in several metals in torsion," Experimental Mechanics, vol. 11, no. 5, pp. 370-374, 1971.

[35] J. Duffy, R. H. Hawley, and R. A. Frantz Jr., "The deformation of lead in torsion at high strain rates," Transactions of the ASME, Journal of Applied Mechanics, vol. 39, no. 3, pp. 651-656, 1972.

[36] J. E. Lawson and T. Nicholas, "The dynamic mechanical behavior of titanium in shear," Journal of the Mechanics and Physics of Solids, vol. 20, no. 2, pp. 65-76, 1972.

[37] T. Nicholas and J. D. Campbell, "Shear-strain-rate effects in a high-strength aluminum alloy," Experimental Mechanics, vol. 12 , no. 10, pp. 441-447, 1972.

[38] J. Lipkin, J. D. Campbell, and J. C. Swearengen, "The effects of strain-rate variations on the flow stress of OFHC copper," Journal of the Mechanics and Physics of Solids, vol. 26, no. 4, pp. 251-268, 1978.

[39] K. T. Ramesh, "On the localization of shearing deformations in tungsten heavy alloys," Mechanics of Materials, vol. 17, no. 2-3, pp. 165-173, 1994.

[40] J. Duffy, J. D. Campbell, and R. H. Hawley, "On the use of a torsional split Hopkinson bar to study rate effects in 11000 aluminum," Journal of Applied Mechanics, vol. 38, no. 1, pp. 83-91, 1971.

[41] X. Nie, R. Prabhu, W. W. Chen, J. M. Caruthers, and T. Weerasooriya, "A Kolsky torsion bar technique for characterization of dynamic shear response of soft materials," Experimental Mechanics, vol. 51, no. 9, pp. 1527-1534, 2011.

[42] Y. Zhou, Q. Fang, L. Chen et al., "A preliminary research on the rotating-wheel TSHB testing system," in Proceedings of 
11th National Conference on Explosion Mechanics, p. 248, Zhuhai, China, 2016.

[43] X. Jiang, Y. Xiao, L. Chen, Q. Fang, and Y. Zhou, "An electromagnetic driven torsional split hopkinson bar," in Proceedings of 2nd International Conference on Impact Loading of Structures and Materials, pp. 103-105, Xi'an, China, May 2018.

[44] J. D. Campbell and A. R. Dowling, "The behaviour of materials subjected to dynamic incremental shear loading," Journal of the Mechanics and Physics of Solids, vol. 18, no. 1, pp. 43-63, 1970.

[45] J. L. Lewis and J. D. Campbell, "The development and use of a torsional Hopkinson-bar apparatus," Experimental Mechanics, vol. 12, no. 11, pp. 520-524, 1972.

[46] M. C. Tsao and J. D. Campbell, Plastic Shear Properties of Metals and Alloys at High Strain Rates, Oxford University, Department of Engineering Science, Oxford, UK, 1973.

[47] A. M. Eleiche and J. D. Campbell, The Influence of Strain-Rate History and Temperature on the Shear Strength of Copper, Titanium and Mild Steel, Oxford University, Department of Engineering Science, Oxford, UK, 1976.

[48] K. A. Hartley, J. Duffy, and R. H. Hawley, "The torsional Kolsky split-Hopkinson bar," ASM Metals Hand, vol. 43, pp. 873-896, 1985.

[49] R. A. Frantz and J. Duffy, "The dynamic stress-strain behavior in torsion of 1100-0 aluminum subjected to a sharp increase in strain rate," Journal of Applied Mechanics, vol. 39, no. 4, pp. 939-945, 1972.

[50] P. E. Senseny, J. Duffy, and R. H. Hawley, "Experiments on strain rate history and temperature effects during the plastic deformation of close-packed metals," Journal of Applied Mechanics, vol. 45, no. 1, pp. 60-66, 1978.

[51] B. J. Claus, X. Nie, B. E. Martin et al., "A side-impact torsion kolsky bar," Experimental Mechanics, vol. 55, no. 7, pp. 1367-1374, 2015.

[52] H. Liu, Mechanics of Materials, vol. 1, Higher Education Press, Beijing, China, 1992, in Chinese.

[53] F. Zhou, L. Wang, and S. Hu, "On the effect of stress nonuniformness in polymer specimen of SHPB tests," Journal of Experimental Mechanics, vol. 7, no. 1, pp. 23-29, 1992, in Chinese.

[54] G. Ravichandran and G. Subhash, "Critical appraisal of limiting strain rates for compression testing of ceramics in a split Hopkinson pressure bar," Journal of the American Ceramic Society, vol. 77, no. 1, pp. 263-267, 1994.

[55] L. M. Yang and V. P. W. Shim, "An analysis of stress uniformity in split Hopkinson bar test specimens," International Journal of Impact Engineering, vol. 31, no. 2, pp. 129-150, 2005.

[56] H. Meng and Q. M. Li, "Correlation between the accuracy of a SHPB test and the stress uniformity based on numerical experiments," International Journal of Impact Engineering, vol. 28, no. 5, pp. 537-555, 2003.

[57] A. Gilat, Torsional Kolsky Bar Testing, ASM International, Materials Park, OH, USA, 2000.

[58] W. W. Chen and B. Song, "Dynamic characterization of soft materials," in Dynamic Failure of Materials and Structures, pp. 1-28, Springer, Berlin, Germany, 2009.

[59] A. M. Eleiche and J. Duffy, "Effects of temperature on the static and dynamic stress-strain characteristics in torsion of 1100-0 aluminum," International Journal of Mechanical Sciences, vol. 17, no. 2, pp. 85-95, 1975.
[60] A. Gilat and C. S. Cheng, "Torsional split Hopkinson bar tests at strain rates above $10^{4} \mathrm{~s}^{-1}$," Experimental Mechanics, vol. 40, no. 1, pp. 54-59, 2000.

[61] K. A. Hartley, J. Duffy, and R. H. Hawley, "Measurement of the temperature profile during shear band formation in steels deforming at high strain rates," Journal of the Mechanics and Physics of Solids, vol. 35, no. 3, pp. 283-301, 1987.

[62] S. Nemat-Nasser and J. B. Isaacs, "Direct measurement of isothermal flow stress of metals at elevated temperatures and high strain rates with application to Ta and Ta-W alloys," Acta Materialia, vol. 45, no. 3, pp. 907-919, 1997.

[63] B. Song and W. Chen, "Loading and unloading split Hopkinson pressure bar pulse-shaping techniques for dynamic hysteretic loops," Experimental Mechanics, vol. 44, no. 6, pp. 622-627, 2004.

[64] R. Chen, F. Lu, Y. Lin et al., "Investigation of the pre-fixed gap in single-pulse loading technique of Hopkinson pressure bars," Chinese Journal of High Pressure Physics, vol. 22, no. 2, pp. 187-191, 2008, in Chinese.

[65] Q. Xue, Y. Bai, and L. Shen, "Modified SHTB and Its Application to Microscopic Study of Thermo-visco-plastic Shear Localization," in Proceedings of the 2nd International Symposium on Intense Dynamic Loading and Its Effects, p. 405, Sichuan University Press, Chengdu, China, December 1992.

[66] Q. Xue, L. T. Shen, and Y. L. Bai, "A modified split Hopkinson torsional bar in studying shear localization," Measurement Science and Technology, vol. 6, no. 11, pp. 1557-1565, 1995.

[67] Q. Xue, L. T. Shen, and Y. L. Bai, "Elimination of loading reverberation in the split Hopkinson torsional bar," Review of Scientific Instruments, vol. 66, no. 11, pp. 5298-5304, 1995.

[68] Q. Xue, L. T. Shen, S. X. Chen et al., “A new split Hopkinson torsional bar for single wave pulse loading," Explosion and Shock Waves, vol. 16, no. 4, pp. 289-297, 1996, in Chinese.

[69] J. L. Lewis and W. Goldsmith, "A biaxial split Hopkinson bar for simultaneous torsion and compression," Review of scientific instruments, vol. 44, no. 7, pp. 811-813, 1973.

[70] H. D. Espinosa, A. Patanella, and M. Fischer, "A novel dynamic friction experiment using a modified Kolsky bar apparatus," Experimental Mechanics, vol. 40, no. 2, pp. 138-153, 2000.

[71] H. Huang and R. Feng, "A study of the dynamic tribological response of closed fracture surface pairs by Kolsky-bar compression-shear experiment," International Journal of Solids and Structures, vol. 41, no. 11-12, pp. 2821-2835, 2004.

[72] S. Rajagopalan and V. Prakash, "A modified torsional Kolsky bar for investigating dynamic friction," Experimental Mechanics, vol. 39, no. 4, pp. 295-303, 1999.

[73] S. Rajagopalan, M. A. Irfan, and V. Prakash, "Novel experimental techniques for investigating time resolved high speed friction," Wear, vol. 225-229, pp. 1222-1237, 1999.

[74] S. Rajagopalan and V. Prakash, "An experimental method to study high speed sliding characteristics during forward and reverse slip," Wear, vol. 249, no. 8, pp. 687-701, 2001.

[75] J. D. Campbell, A. M. Eleiche, and M. C. Tsao, "Strength of metals and alloys at high strains and strain rates," in Fundamental Aspects of Structural Alloy Design, pp. 545-563, Springer, Berlin, Germany, 1977.

[76] A. M. Eleiche, "Strain-rate history and temperature effects on the torsional-shear behavior of a mild steel," Experimental Mechanics, vol. 21, no. 8, pp. 285-294, 1981.

[77] J. L. Chiddister and L. E. Malvern, "Compression-impact testing of aluminum at elevated temperatures," Experimental Mechanics, vol. 3, no. 4, pp. 81-90, 1963. 
[78] U. S. Lindholm and L. M. Yeakley, "High strain-rate testing: tension and compression," Experimental Mechanics, vol. 8, no. 1, pp. 1-9, 1968.

[79] Z. Ruan, L. Chen, and Q. Fang, "Numerical investigation into dynamic responses of RC columns subjected for fire and blast," Journal of Loss Prevention in the Process Industries, vol. 34, pp. 10-21, 2015.

[80] L. Chen, Q. Fang, X. Jiang, Z. Ruan, and J. Hong, "Combined effects of high temperature and high strain rate on normal weight concrete," International Journal of Impact Engineering, vol. 86, pp. 40-56, 2015.

[81] X. Yu, L. Chen, Q. Fang, Z. Ruan, J. Hong, and H. Xiang, “A concrete constitutive model considering coupled effects of high temperature and high strain rate," International Journal of Impact Engineering, vol. 101, pp. 66-77, 2017.

[82] A. Gilat and X. Wu, "Elevated temperature testing with the torsional split Hopkinson bar," Experimental Mechanics, vol. 34, no. 2, pp. 166-170, 1994.

[83] W. Chen and G. Ravichandran, "Dynamic compressive failure of a glass ceramic under lateral confinement," Journal of the Mechanics and Physics of Solids, vol. 45, no. 8, pp. 1303-1328, 1997.

[84] D. J. Frew, M. J. Forrestal, and W. Chen, “A split Hopkinson pressure bar technique to determine compressive stress-strain data for rock materials," Experimental Mechanics, vol. 41, no. 1, pp. 40-46, 2001.

[85] B. Song and W. Chen, "Dynamic compressive behavior of EPDM rubber under nearly uniaxial strain conditions," Journal of Engineering Materials and Technology, vol. 126, no. 2, pp. 213-217, 2004.

[86] J. L. Yu, J. R. Li, and S. S. Hu, "Strain-rate effect and microstructural optimization of cellular metals," Mechanics of Materials, vol. 38, no. 1-2, pp. 160-170, 2006.

[87] B. Song, W. Chen, Y. Ge, and T. Weerasooriya, "Dynamic and quasi-static compressive response of porcine muscle," Journal of Biomechanics, vol. 40, no. 13, pp. 2999-3005, 2007.

[88] D. L. Grote, S. W. Park, and M. Zhou, "Dynamic behavior of concrete at high strain rates and pressures: I. experimental characterization," International Journal of Impact Engineering, vol. 25, no. 9, pp. 869-886, 2001.

[89] Y. Luo, L. Lv, B. Sun, Y. Qiu, and B. Gu, "Transverse impact behavior and energy absorption of three-dimensional orthogonal hybrid woven composites," Composite Structures, vol. 81, no. 2, pp. 202-209, 2007. 


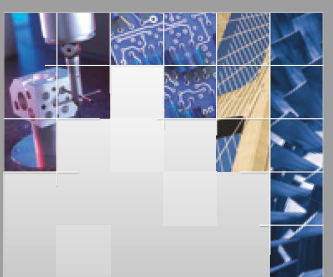

\section{Enfincering}
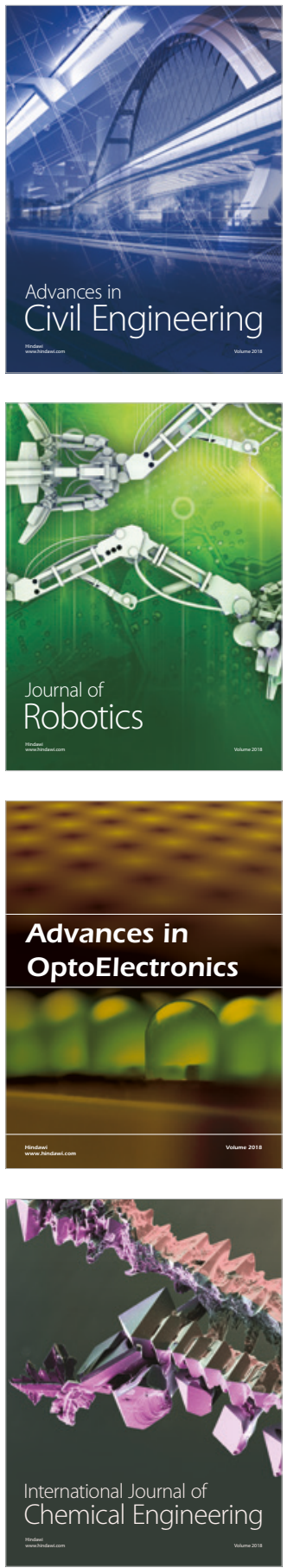

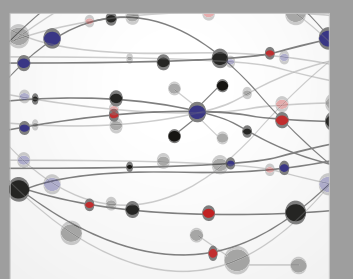

\section{Rotating \\ Machinery}

The Scientific World Journal

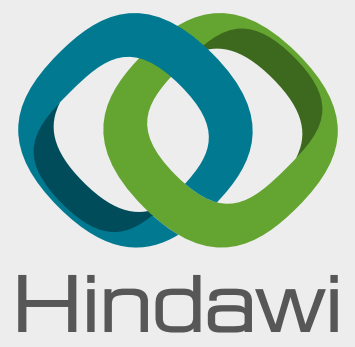

Submit your manuscripts at

www.hindawi.com
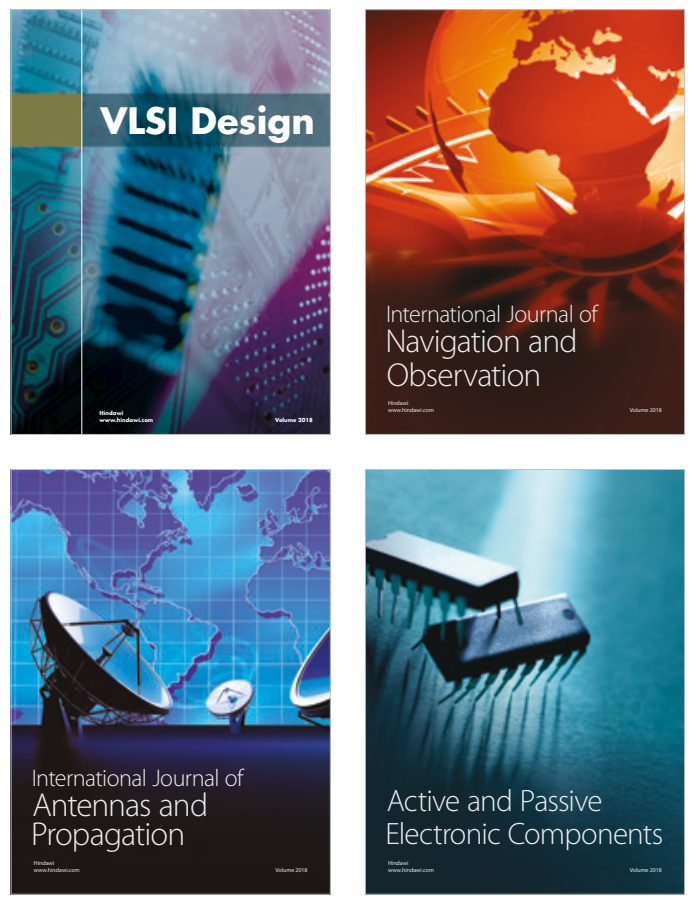
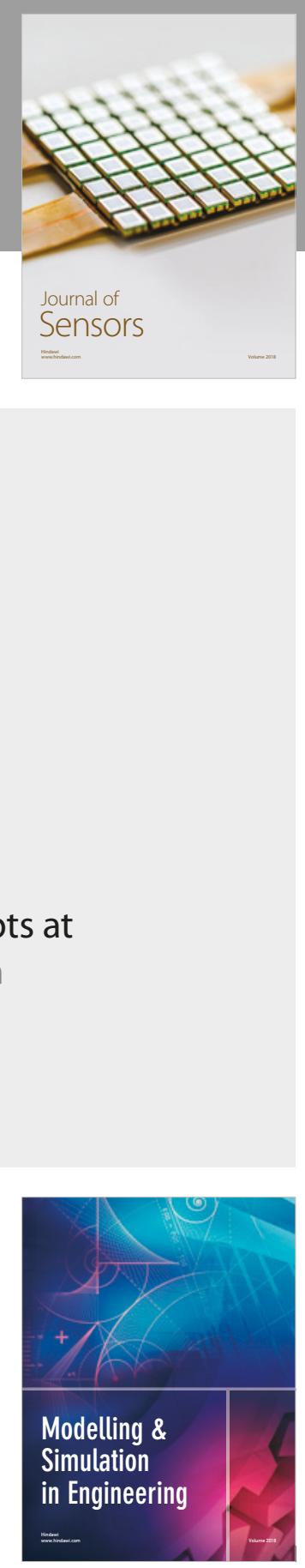

\section{Advances \\ Multimedia}
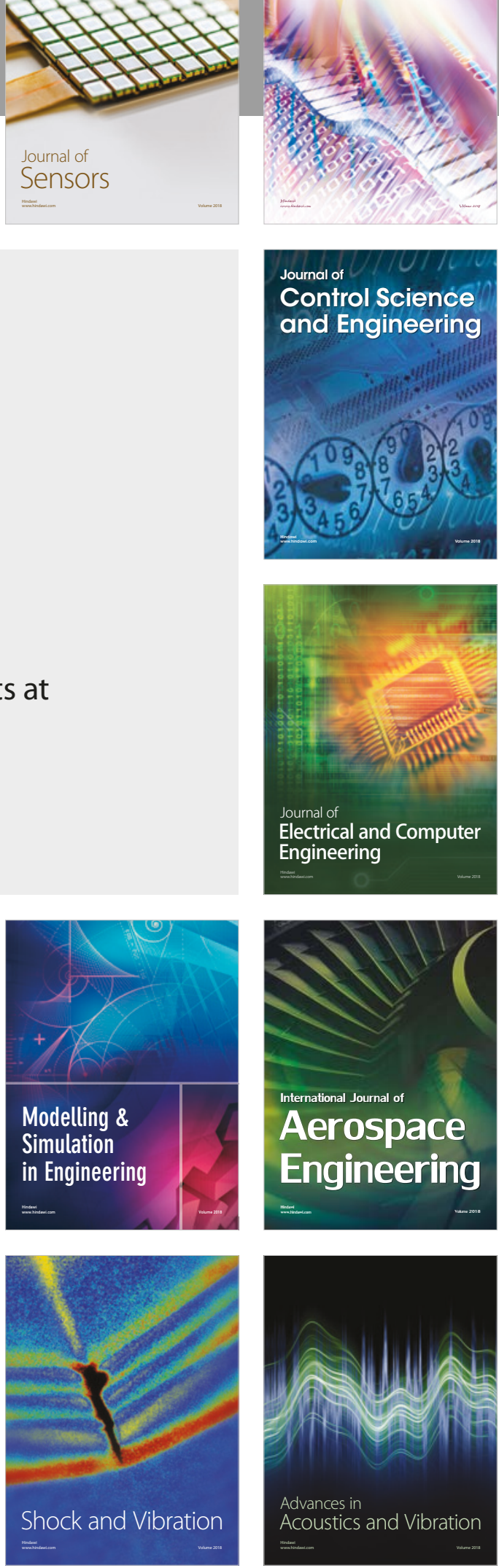